\title{
Environmentally Induced Epigenetic Transgenerational Inheritance of Ovarian Disease
}

\author{
Eric Nilsson, Ginger Larsen, Mohan Manikkam, Carlos Guerrero-Bosagna, Marina I. Savenkova, \\ Michael K. Skinner*
}

School of Biological Sciences, Center for Reproductive Biology, Washington State University, Pullman, Washington, United States of America

\begin{abstract}
The actions of environmental toxicants and relevant mixtures in promoting the epigenetic transgenerational inheritance of ovarian disease was investigated with the use of a fungicide, a pesticide mixture, a plastic mixture, dioxin and a hydrocarbon mixture. After transient exposure of an F0 gestating female rat during embryonic gonadal sex determination, the F1 and F3 generation progeny adult onset ovarian disease was assessed. Transgenerational disease phenotypes observed included an increase in cysts resembling human polycystic ovarian disease (PCO) and a decrease in the ovarian primordial follicle pool size resembling primary ovarian insufficiency (POI). The F3 generation granulosa cells were isolated and found to have a transgenerational effect on the transcriptome and epigenome (differential DNA methylation). Epigenetic biomarkers for environmental exposure and associated gene networks were identified. Epigenetic transgenerational inheritance of ovarian disease states was induced by all the different classes of environmental compounds, suggesting a role of environmental epigenetics in ovarian disease etiology.
\end{abstract}

Citation: Nilsson E, Larsen G, Manikkam M, Guerrero-Bosagna C, Savenkova MI, et al. (2012) Environmentally Induced Epigenetic Transgenerational Inheritance of Ovarian Disease. PLoS ONE 7(5): e36129. doi:10.1371/journal.pone.0036129

Editor: Toshi Shioda, Massachusetts General Hospital, United States of America

Received December 22, 2011; Accepted March 30, 2012; Published May 3, 2012

Copyright: (C) 2012 Nilsson et al. This is an open-access article distributed under the terms of the Creative Commons Attribution License, which permits unrestricted use, distribution, and reproduction in any medium, provided the original author and source are credited.

Funding: The research was supported by Department of Defense and National Institutes of Health. National Institute of Environmental Health Sciences support to MKS. The funders had no role in study design, data collection and analysis, decision to publish, or preparation of the manuscript.

Competing Interests: The authors have declared that no competing interests exist.

* E-mail: skinner@wsu.edu

\section{Introduction}

Environmental exposures during fetal and early postnatal development can lead to an increased incidence of later life adult-onset diseases $[1,2,3,4]$. Such environmental factors include nutritional abnormalities, stress and exposure to toxicants. Examples include fetal exposures to plasticizers such as bisphenol A leading to immune abnormalities [5], maternal smoking leading to increased pulmonary disease in adulthood [6], nutrition defects leading to hypertension in offspring $[7,8]$ and therapeutic drug exposure leading to vascular defects [9]. In addition to these direct effects of early life exposure on adult onset disease, environmental factors have been shown to affect the next F2 generation $[8,10,11,12]$. The subsequent generations transgenerational inheritance of epigenetic changes in the genome now provides an additional molecular mechanism, along with classic induction of genetic mutations, for the germ line transmission of environmentally induced phenotypic change $[2,13,14]$.

Effects on the F1 and F2 generation can be due to direct multigenerational exposure to the environmental factor [13,15]. If a gestating female is defined as the F0 founder generation, then the fetal offspring are the F1 generation, and the germ cells present in those developing fetuses will eventually become the eggs or sperm that would form the F2 generation. An environmental exposure of an F0 generation gestating female directly exposes both the F1 generation fetuses and the germ cells present in those fetuses that will generate the F2 generation $[2,13,15]$. The subsequent F3 generation would be the first generation that would not have been directly exposed to the environmental factor. Therefore, effects on the F1 and F2 generation can be due to direct exposure and so should be considered multigenerational effects [13]. In contrast, a transgenerational effect following exposure of a F0 generation gestating female is defined as an effect seen in the F3 or later generations [15]. Transgenerational phenomena by definition do not involve direct exposure and have been shown to involve epigenetic changes induced in the germ line $[16,17,18,19]$.

The initial report of epigenetic transgenerational inheritance of adult onset disease was from gestating female rats exposed to the fungicide vinclozolin, in which F3 generation male offspring showed defects in sperm production [16,20]. Transgenerational effects have also been reported after exposure of gestating rats to bisphenol A (BPA), where decreased fertility was seen in the F3 generation males [21]. Decreased fertility was also seen in F3 and $\mathrm{F} 4$ generation female mice after the gestating F0 generation was exposed to dioxin [22]. Similarly in mice, male F3 generation offspring showed changes in the methylation pattern of imprinted genes in sperm following exposure of the gestating F0 generation female to the agricultural fungicide vinclozolin [23]. Recently, a number of different exposures to environmental toxicants including BPA, phthalates, dioxin, pesticide, DEET and jet fuel hydrocarbons were found to promote epigenetic alterations in sperm and transgenerational inheritance of reproduction defects [19]. Therefore, a number of different environmental toxicants and other factors such as nutrition [4] can promote epigenetic transgenerational inheritance of adult onset disease.

In women, adult-onset diseases of the ovary that can dramatically affect fertility are primary ovarian insufficiency and polycystic ovarian disease. Primary ovarian insufficiency (POI) is 
characterized by a significant reduction in the primordial follicle pool of oocytes (eggs) that appears intrinsic to the ovary, and induction of menopause prior to age 40 [24]. This is associated with decreased estrogen and elevated gonadotropin levels in the blood. POI affects about $1 \%$ of women [25,26,27]. A reduced primordial follicle pool size correlated with POI has been shown in sheep and primates to also associate with polycystic ovarian disease $[28,29]$. POI is often thought to have a genetic basis since chromosomal abnormalities and single gene mutations are associated with a percentage of POI cases. However, only a minority (4-20\%) of human cases can be ascribed a genetic basis [26,27,30,31,32,33,34,35,36].

Polycystic ovarian (PCO) disease or polycystic ovary syndrome (PCOS) is a common endocrine disorder that affects $6-18 \%$ of women $[37,38,39,40,41,42]$. It is characterized by infrequent ovulation or anovulation, high androgen levels in the blood, and the presence of multiple persistent ovarian cysts [43]. PCOS patients often show insulin resistance and a heightened risk for diabetes [44]. Current thought on the etiologies that lead to development of PCOS is that there are both genetic and environmental causal factors. A genetic predisposition in an individual may combine with an early-life environmental impact such as fetal stress or increased androgens in utero and lead to development of PCOS in adulthood $[44,45,46]$. Fetal or early postnatal exposure to androgens (e.g. di-hydrotestosterone) has been shown to promote $\mathrm{PCO}$ and associated clinical parameters (e.g. metabolic abnormalities, adiposity and endocrine abnormalities) in rats, mice and sheep $[47,48,49]$. Therefore, the rodent PCO model has many of the same clinical correlations that are seen with PCOS in humans. Sequence variations in several genes have been associated with PCOS [46,50,51], although at very low frequency and none are highly predictive. Epigenetic abnormalities such as those associated with non-random X-chromosome inactivation have also been linked to PCOS [51,52,53,54]. Several diseases are now known to have an important epigenetic component, such as allergies [55], hepatic cancer [56], gastric cancer [57], asthma [58], colorectal cancer [59], prostate cancer [60], HIV latency [61] and psychiatric disorders [62]. The current study was designed to investigate the role of environmental epigenetics in ovarian disease.

A rat model is used to evaluate whether adult onset ovarian diseases are induced transgenerationally after exposure of a gestating F0 generation female to known environmental toxicants. The exposures are during days 8-15 of fetal development, which is the time of gonadal sex determination. The exposure compounds were: 1) Vinclozolin, an agricultural fungicide previously shown to cause transgenerational epigenetic disease [13,20,23]; 2) A mixture of permethrin, the most commonly used human insecticide shown to have minor toxicologic effects in mammals [63] and DEET, an insect repellent reported to have negligible toxic effects [63]; 3) A plastic mixture of bisphenol A (BPA), dibulylphthalate (DBP) and bis(2-ethylexyl)phthalate (DEHP), all plasticizer chemicals that commonly appear together from plastics with in vitro and in vivo toxic effects [64]; 4) Dioxin (TCDD), a by-product of some commercial chemical syntheses that has been shown to induce adult-onset diseases including premature acyclicity [65,66]; and 5) Jet fuel (JP8), a hydrocarbon mixture (i.e. C3->C20) often used for dust control on road surfaces, with known toxicologic effects $[67,68]$, but is not known to induce reproductive defects [69]. The United States Department of Defense assisted in the selection of these toxicants and mixtures due to their relevance for exposures anticipated for military personnel. The plastic mixture included the three common toxicants present in heated bottled water, the pesticide mixture is the most common used in humans and the hydrocarbon mixture (i.e. jet fuel JP8) is commonly used in dust control on road surfaces. All of the above environmental toxicants have been implicated in inducing transgenerational disease phenotypes $[13,19,21,22]$. The current study used pharmacological doses and administration to assess potential transgenerational actions on ovarian disease and should not be considered a risk assessment analysis. Future studies are now needed to do environmental risk assessment, based on the observations of the current studies.

The adult F3 generation females from each exposure lineage group were examined for the incidence of ovarian diseases similar to primary ovarian insufficiency and polycystic ovarian disease. The human ovarian diseases POI and PCOS have numerous other clinical conditions associated with them such as endocrine abnormalities and glucose intolerance. Therefore, the rat ovarian abnormalities/disease cannot be directly correlated to the clinical aspects of human ovarian disease, but do share the majority of morphological changes. In order to gain insight into possible cellular and molecular mechanisms involved in ovarian disease development, the granulosa cells from F3 generation vinclozolin and control lineage animals were evaluated for changes to their transcriptome and epigenome (DNA methylation pattern). All the primary cell types of an ovarian follicle such as the oocyte, theca cells and granulosa cells are anticipated to develop a transgenerationally altered transcriptome and epigenome [14], and so will participate in the adult onset disease development. The granulosa cell was selected to provide the proof of concept that such an alteration in genome activity could develop. Future studies will investigate the other cell types. The capacity of vinclozolin to directly induce oocyte loss in ovaries was also examined to clarify how $\mathrm{F} 1$ generation effects may develop. Observations demonstrate that the environmental toxicants examined induced transgenerational ovarian adult-onset disease, and suggest that primary ovarian insufficiency and polycystic ovarian disease can have an epigenetic transgenerational etiology.

\section{Results}

\section{Transgenerational Ovarian Disease}

Gestating female rats, designated as F0 generation animals, were treated by intraperitoneal injection daily from E8 (post conception gestational day 8) through E14. The F0 generation female rats received one of five different treatments as described in Methods: Vinclozolin, Pesticide (includes permethrin and DEET), Plastics (includes BPA, DBP and DEHP), Low-dose Plastics $(50 \%$ of Plastics dose), Dioxin, Hydrocarbon (Jet fuel JP8), or DMSO vehicle as Control. The F1 generation offspring were bred to others of the same treatment group to produce an F2 generation, and F2 generation animals were similarly bred to produce an F3 generation (see Methods). No sibling or cousin breedings were used to avoid any inbreeding artifacts. Only the original F0 generation gestating female rats received the treatment exposures. Female rats from the F1 and F3 generations were kept until one year of age and then sacrificed. Ovaries were removed, fixed, sectioned and stained for histologic examination.

The number of oocytes (i.e. eggs) present in the ovaries was determined by counting follicles. Each ovarian follicle is composed of an oocyte surrounded by a layer of granulosa cells, a basement membrane and outer layers of thecal cells. Primordial follicles are in an arrested state of development and contain a single layer of squamous flattened granulosa cells. Developing follicles have multiple layers of proliferating granulosa cells and an increase in the oocyte diameter. Later in follicle development a fluid-filled antrum forms $[70,71,72,73]$. In the F1 and F3 generation, ovarian 
morphological evaluation and counts were performed to determine the number of primordial follicles, pre-antral developing follicles and antral developing follicles as described in Methods [74].

In F1 generation ovaries there was a marked and statistically significant $(p<0.001)$ reduction in the number of primordial follicles in all exposure groups compared to ovaries from the vehicle-treated control lineage (Fig. 1). This indicates that the female fetuses exposed to these compounds during gonadal sex determination all have a decrease in their resting pool of primordial follicles. There was no change in the number of preantral developing follicles or antral developing follicles, except in the case of females of the vinclozolin-treated lineage. F1 vinclozolin group ovaries had significantly $(\mathrm{p}<0.05)$ fewer preantral developing follicles compared to controls (Fig. 1). This effect of the exposures on the Fl generation is attributed to direct fetal ovarian exposure to the treatments.

Since treatment with environmental toxicants resulted in fewer oocytes being present in $\mathrm{F} 1$ generation adult ovaries compared to controls, an experiment was performed to assess if vinclozolin could act directly on ovaries to reduce oocyte number. Ovaries from four-day old rats, containing predominately primordial follicles, were placed into a whole-ovary culture system (see Methods) and treated in vitro for ten days with varying concentrations of vinclozolin. Treatment with $500 \mu \mathrm{M}$ vinclozolin did result in a decrease $(\mathrm{p}<0.05)$ in oocyte number compared to controls (Fig. 2). The $200 \mu \mathrm{M}$ and lower doses of vinclozolin did not significantly reduce oocyte number. Therefore, direct actions of vinclozolin on the F1 generation fetal gonad have the potential to reduce follicle numbers if the dose is sufficient.
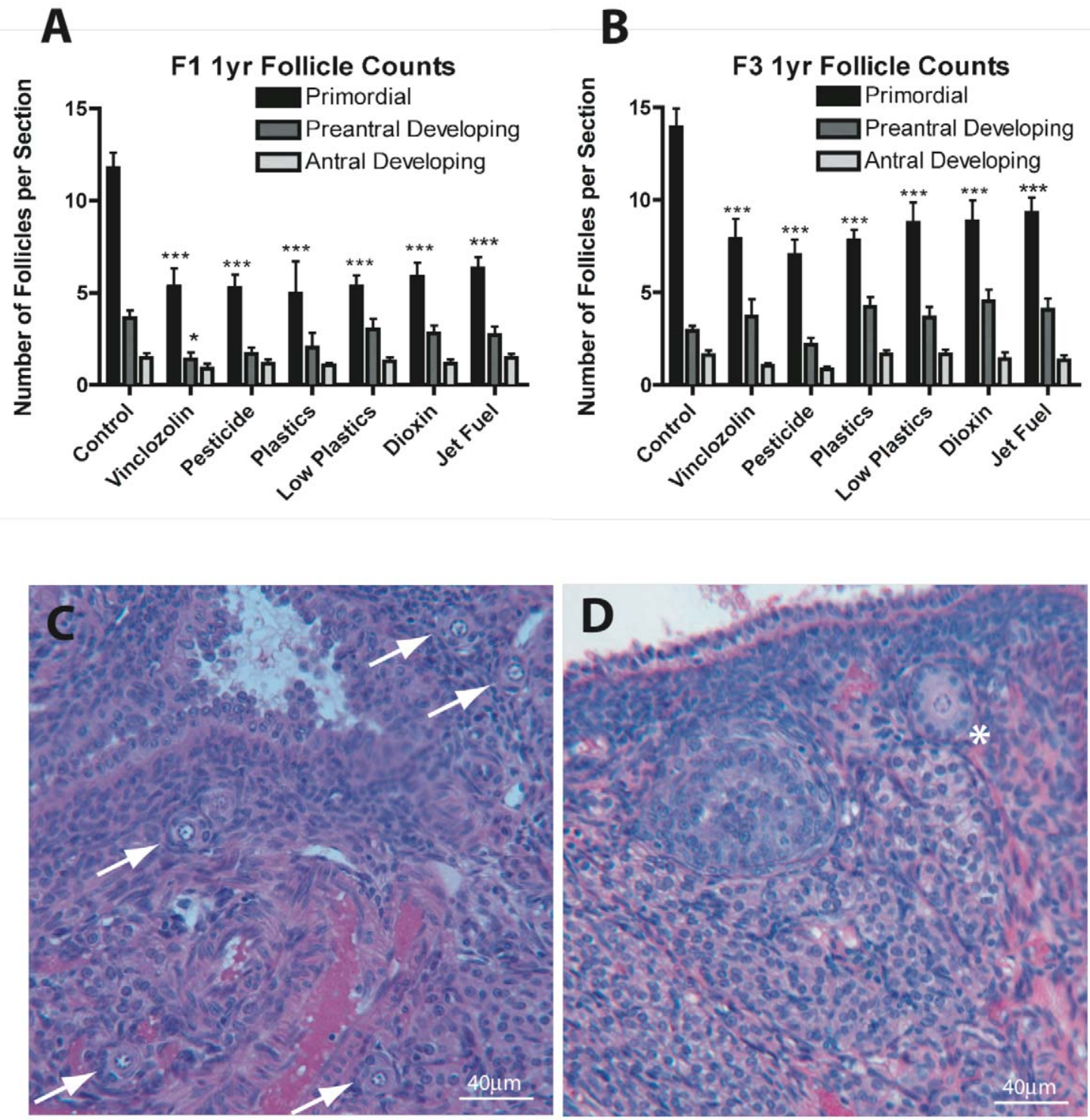

Figure 1. Follicle numbers and development. A) Number of primordial, preantral, and antral follicles per section in $\mathrm{F} 1 \mathrm{generation}$ ovaries. $\mathrm{N}=9$ animals per treatment group. B) Number of follicles per section in F3 generation ovaries. $\mathrm{N}=9$ animals per treatment group. Asterisks indicate groups significantly $\left.{ }^{*} \mathrm{p} \leq 0.05,{ }^{* * *} \mathrm{p} \leq 0.005\right)$ different than controls of their own follicle type by ANOVA followed by Dunnet's post-hoc test. C) H and E stained section of F3 generation control ovary showing several primordial follicles (arrows). D) $\mathrm{H}$ and E stained section of F3 generation vinclozolin lineage ovary without visible primordial follicles. Asterisk indicates a developing secondary follicle. Scale bar $=40 \mu \mathrm{m}$. doi:10.1371/journal.pone.0036129.g001 
Total oocytes

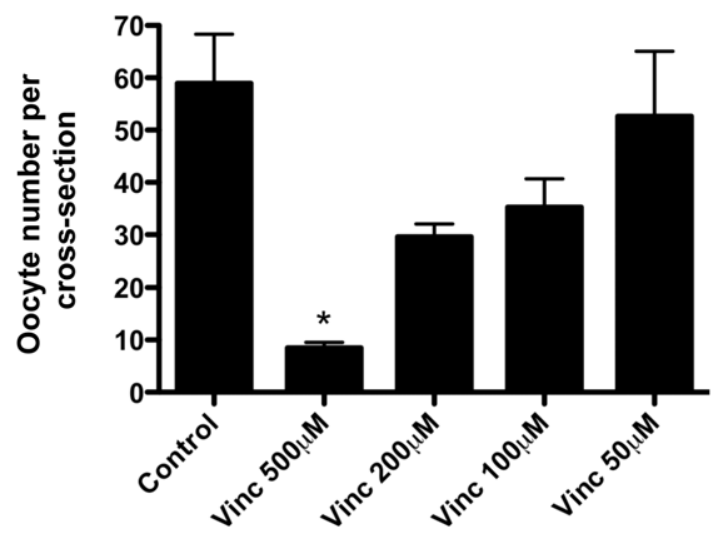

Figure 2. Number of oocytes per ovarian cross-section in ovaries taken from 4-day old rats and cultured whole for 10 days in the presence of different concentrations of vinclozolin. Data are from five different experiments performed in replicate. Asterisks indicate groups significantly $\left({ }^{*} p \leq 0.05\right)$ different than controls by ANOVA followed by Dunnet's post-hoc test. doi:10.1371/journal.pone.0036129.g002

In F3 generation ovaries, similarly to F1 females, there was a significant $(\mathrm{p}<0.001)$ reduction in the number of primordial follicles in all treatment groups, compared to controls (Fig. 1). Since none of these F3 generation animals were themselves exposed to the treatment compounds, this reduction in oocyte number is a transgenerational effect. There was no change in the number of preantral developing or antral developing follicles for any exposure lineage group compared to control lineage animals. Therefore, all the exposure groups examined induced a significant transgenerational decline in the primordial follicle pool size. For the purposes of this study, the ovaries of an animal were classified as having "disease" if the ovary primordial follicle numbers were $\geq 2$ standard deviations less than that seen in controls. The incidence of the follicle pool disease was $33-60 \%$ across treatment groups.

Polycystic ovarian (PCO) disease is a common disease in humans, so ovaries from $\mathrm{F} 1$ and $\mathrm{F} 3$ generation animals were evaluated for the presence of cystic structures. Ovarian cysts were defined and categorized as either small or large cysts, as described in Methods. Interestingly, an increase in the number of both small and large cysts were seen most often in F3 generation ovaries from exposure lineages, rather than in F1 generation ovaries (Fig. 3). An increase $(\mathrm{p}<0.01)$ in small cysts was seen in all F3 generation treatment groups, compared to controls. However, in the F1 generation only the low-dose plastics, jet fuel hydrocarbons and vinclozolin lineage ovaries showed an increase $(p<0.05)$ in small cysts. An increase in large cysts was observed in the F3 generation ovaries of the vinclozolin, pesticide, low-dose plastics and jet fuel treatment groups (Fig. 3). However, in F1 generation ovaries only the low-dose plastics showed an increase in the incidence of large cysts compared to controls. These results indicate that development of ovarian cysts occurs more often in the F3 generation, which demonstrates a transgenerational effect of the toxicant exposures. The large cysts observed in these ovaries often were lined with a sporadic single layer of epithelial granulosa cells and were surrounded by a band of theca cells (Fig. 3F). This is consistent with these large cysts being derived from antral follicles. However, some large cysts and associated cells were morphologically identified as being from corpora lutea [19]. These luteal cysts were present frequently in the F3 generation jet fuel hydrocarbon exposure lineage ovaries, Figure 3.

The number of healthy-looking large antral follicles was not found to be different between exposure and control groups in either the F1 or F3 generation ovaries (Fig. 4A \& B). The exception was that there were significantly fewer $(\mathrm{p}<0.05)$ large antral follicles in the F3 pesticide-lineage ovaries. Therefore, the antral follicle development process appears relatively normal in the $\mathrm{F} 1$ and F3 generation females independent of exposure lineage.

Previous studies have demonstrated that negligible endocrine abnormalities are detected in 120-day-old F3 generation female rats following exposure to any of the toxicants studied $[19,75]$. PCO has previously been associated with an increase in androgen serum levels which is due to the highly steroidogenic theca cells of the cysts. Theca cells primarily produce androstenedione so the serum androstenedione levels in the F3 generation 1-year-old females were examined. Preliminary studies show that the F3 generation control lineage had $47 \pm 3 \mathrm{pg} / \mathrm{mL}$ and the vinclozolin lineage had $177 \pm 82 \mathrm{pg} / \mathrm{mL}$ serum androstenedione. Therefore, the androgen levels were increased in the F3 generation vinclozolin lineage females that had the PCO disease. This increase in androgen levels requires further investigation as do the other associated clinical conditions of glucose intolerance, abnormal adiposity and hyperinsulemia.

\section{Transgenerational Granulosa Cell Transcriptome}

Previous studies demonstrated the vinclozolin induced epigenetic transgenerational inheritance of adult onset disease involving epigenetic modifications of the sperm [16] and heritable phenotypes through the paternal lineage [20]. The only cell that can transmit an altered epigenome between generations is the germline [13], however, all the cells derived from this sperm will have an altered epigenome transcriptome [14]. In order to see if transgenerational changes in gene expression are apparent in ovarian follicle cells of the exposure lineage females, the transcriptomes of granulosa cells from control and vinclozolin lineage ovaries were compared. Granulosa cells were collected from pre-ovulatory follicles of five-month old F3 generation vinclozolin and control lineage ovaries as described in Methods. Messenger RNA was isolated from the granulosa cells of each animal $(\mathrm{n}=24)$ and RNA from four animals of the same treatment group were pooled to create three different pooled samples from each of the two treatment groups. Three F3 generation vinclozolin-lineage and three control-lineage mRNA pooled samples were used in a microarray analysis as described in Methods to evaluate alterations in gene expression. The analysis demonstrated that 523 genes were differentially expressed between control and vinclozolin lineage F3 generation granulosa cells (Table S1). The number of differentially expressed genes in each of several functional gene categories is shown in Figure 5 with the number of up-regulated and down-regulated genes indicated. Many of the differentially expressed genes were identified as contributing to metabolism or signaling processes. The complete list of differentially expressed genes is functionally categorized and presented in Table S1.

A table of cellular pathways and processes impacted by the genes differentially expressed in vinclozolin lineage F3 generation granulosa cells is presented (Table 1). In Figure S2, two of the more heavily impacted cellular pathways are shown, PPAR signaling and steroid biosynthesis, with the differentially expressed genes highlighted. These data indicate that gene expression is altered transgenerationally in granulosa cells and that specific physiological processes may be affected by these changes. Additional bioinformatic analyses examined the functional rela- 
A
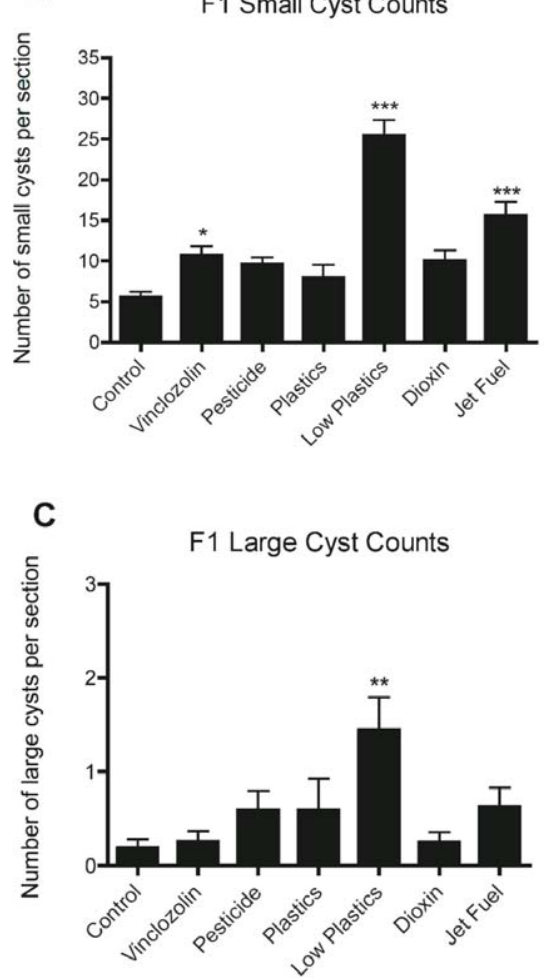

B
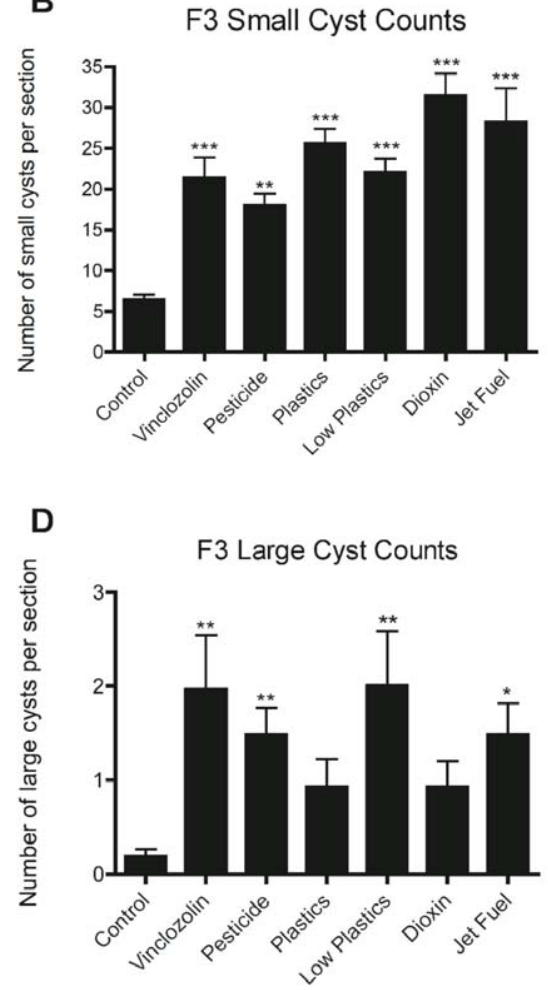

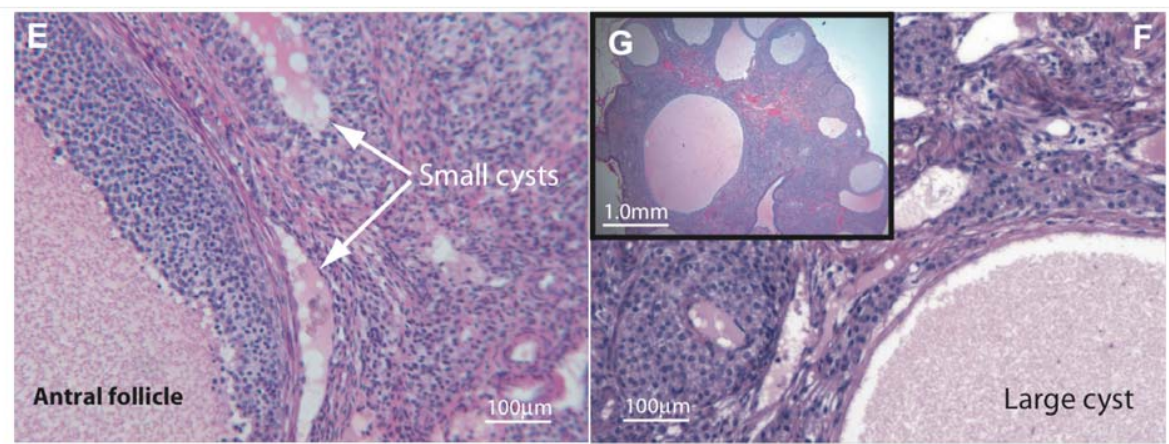

Figure 3. Ovarian cysts. Number of small $(50-250 \mu \mathrm{m})$ cysts per section in F1 (A) and F3 (B) generation ovaries. Number of large ( $>250 \mu \mathrm{m})$ cysts per section in $\mathrm{F} 1$ (C) and $\mathrm{F} 3$ (D) generation ovaries. $N=9$ animals per treatment group. Asterisks indicate groups significantly $\left({ }^{*} p \leq 0.05,{ }^{* *} p \leq 0.01\right.$, *** $\mathrm{p} \leq 0.005$ ) different than controls by ANOVA followed by Dunnet's post-hoc test. E) $\mathrm{H}$ and $\mathrm{E}$ stained section of $\mathrm{F} 3$ ovary showing small cysts. F) $\mathrm{H}$ and E stained F3 ovary showing a large cyst. G) Expanded view of small and large cysts. doi:10.1371/journal.pone.0036129.g003

tionships among the F3 generation differentially expressed genes identified. An unbiased literature based network analysis was performed as described in Methods to determine which genes are functionally linked with respect to binding, signaling or regulation. This created a gene network of direct connections as shown in Figure 6. Some genes show significant functional connections to others, such as ESR1, MMP2 and CXCL12. Such highly connected genes may play important regulatory roles in these F3 generation granulosa cells and in the development of ovarian disease states. Therefore, a transgenerational change in the granulosa cell transcriptome was identified that may be in part a causal factor in the molecular etiology of the transgenerational ovarian disease. Further analysis of the 523 genes with transgenerational alterations in gene expression identified previously known genes involved in ovarian disease and more specifically polycystic ovarian disease. A total of 30 genes were found to be related to ovarian disease and 5 directly related to PCO disease, Figure 7. Therefore, genes known to have a relationship with PCO and ovarian disease were shown to have altered expression. The potential role of the transgenerational change in the granulosa cell epigenome to promote this transcriptome alteration is described below.

\section{Transgenerational Granulosa Cell Epigenome}

As previously described $[13,16,20]$, an epigenetic transgenerational alteration of the sperm in vinclozolin lineage F3 generation animals can promote a transgenerational change in the epigenome unique to each cell type in all somatic cells derived from this germ line $[14,76]$. The F3 generation vinclozolin lineage alterations in differentially DNA methylated regions (DMR) in the granulosa cells was investigated. For this, a methylated DNA immunoprecipitation (MeDIP) procedure was used, followed by comparative 

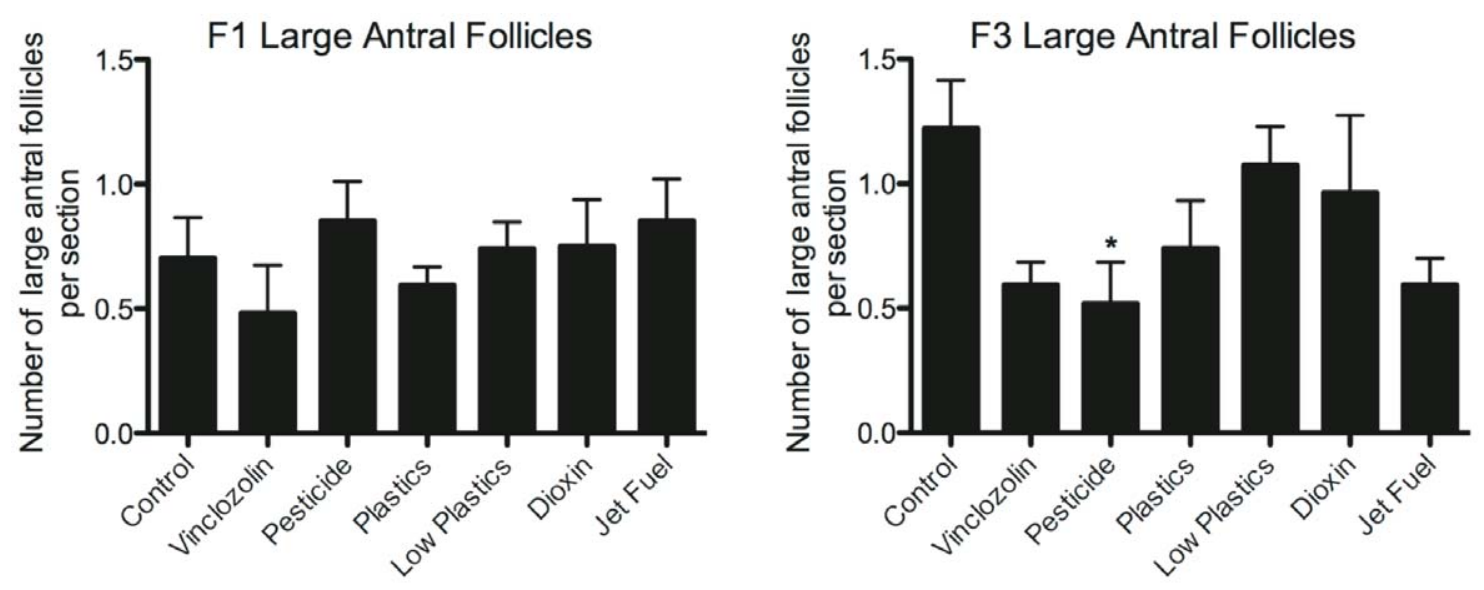

Figure 4. Large antral follicles. A) Number of large antral follicles per section in F1 generation ovaries. B) Number of large antral follicles per section in $\mathrm{F} 3$ generation ovaries. $\mathrm{N}=9$ animals per treatment group. Asterisks indicate groups significantly ( $\left.{ }^{*} \mathrm{p} \leq 0.05\right)$ different than controls by ANOVA followed by Dunnet's post-hoc test.

doi:10.1371/journal.pone.0036129.g004

hybridization on a genome wide promoter tiling array (Chip), termed an MeDIP-Chip assay, as previously described [16]. The MeDIP-Chip analysis of the differential DNA methylation between control and vinclozolin lineage F3 generation granulosa cells identified 43 DMR with a statistical significance $\mathrm{p}>10^{-7}$, Table 2. The chromosomal locations of all the DMR are presented in Figure 8 and indicates most autosomes are involved. A comparison of the 43 DMR identified with the 523 differentially expressed granulosa cell genes demonstrated only 1 gene promoter with overlap (Plekhm1). Analysis of the probability for a random overlap between the $43 \mathrm{DMR}$ and the 523 differentially expressed genes indicated that an overlap of 1.47 genes would be expected.
Therefore, the one gene overlap is likely not significant. The vast majority of differentially expressed genes did not have a DMR present in their promoters. Further analysis using statistically significant over represented clusters of differentially expressed genes identified 26 clusters from 2-5 Mbase size that had 4 to 9 genes each, Table S2. An overlap of these regulated gene clusters with the DMR identified 3 overlapped clusters, Figure 8. These 25 Mbase regions we refer to as potential Epigenetic Control Regions (ECR). The hypothesis is that the epigenetic regulatory site (e.g. DMR) regulates distally the expression of genes within this ECR. This is likely mediated through non-coding RNA, similar to what is seen for imprinting control regions (ICR)

F3 Generation Granulosa Cell Transcriptome Alterations

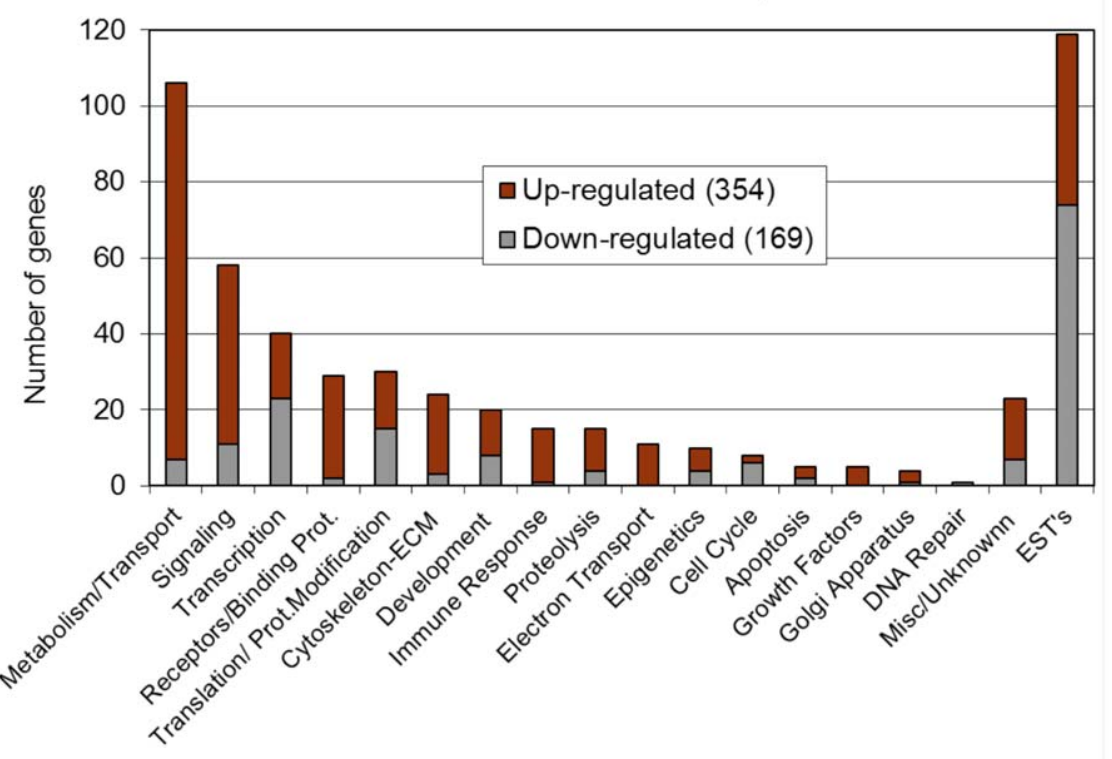

Figure 5. Number of genes with mRNA expression levels significantly different between Control and Vinclozolin-lineage F3 generation granulosa cells. Genes are placed into functional categories. doi:10.1371/journal.pone.0036129.g005 
Table 1. Physiological Pathway Enrichment.

\begin{tabular}{|c|c|c|}
\hline Pathway Name & $\begin{array}{l}\text { \# Input Genes } \\
\text { in Pathway }\end{array}$ & $\begin{array}{l}\text { Impact } \\
\text { Factor** }\end{array}$ \\
\hline PPAR signaling pathway & 11 & 17.0 \\
\hline Phagosome & 11 & \\
\hline Cell adhesion molecules (CAMs) & 10 & 10.3 \\
\hline Endocytosis & 10 & \\
\hline Steroid biosynthesis & 9 & \\
\hline Peroxisome & 8 & \\
\hline Antigen processing and presentation & 8 & 38.5 \\
\hline Leukocyte transendothelial migration & 8 & 8.9 \\
\hline Fatty acid metabolism & 6 & \\
\hline Valine, leucine and isoleucine degradation & 6 & \\
\hline Spliceosome & 6 & \\
\hline Lysosome & 6 & \\
\hline Fc gamma R-mediated phagocytosis & 6 & \\
\hline Regulation of actin cytoskeleton & 6 & 4.7 \\
\hline Cysteine and methionine metabolism & 5 & \\
\hline Glutathione metabolism & 5 & \\
\hline Glycerophospholipid metabolism & 5 & \\
\hline Biosynthesis of unsaturated fatty acids & 5 & 10.2 \\
\hline MAPK signaling pathway & 5 & 2.9 \\
\hline Neuroactive ligand-receptor interaction & 5 & 1.5 \\
\hline Focal adhesion & 5 & 5.0 \\
\hline Purine metabolism & 4 & \\
\hline Lysine degradation & 4 & \\
\hline Phenylalanine metabolism & 4 & \\
\hline Sphingolipid metabolism & 4 & \\
\hline Base excision repair & 4 & 7.7 \\
\hline Calcium signaling pathway & 4 & 4.4 \\
\hline Adherens junction & 4 & 7.7 \\
\hline Tight junction & 4 & 5.7 \\
\hline Complement and coagulation cascades & 4 & 7.2 \\
\hline Jak-STAT signaling pathway & 4 & 3.8 \\
\hline Pathways in cancer & 4 & 2.3 \\
\hline \multicolumn{3}{|c|}{$\begin{array}{l}\text { Using } 523 \text { differentially expressed genes (F3 vinclozolin vs. control granulosa) in } \\
\text { KEGG pathway database. } \\
{ }^{* *} \text { Calculated by Pathway Express to estimate importance of these genes to } \\
\text { pathway. } \\
\text { doi:10.1371/journal.pone. } 0036129 . t 001\end{array}$} \\
\hline
\end{tabular}

previously identified [77]. A limited number of long non-coding RNA in the rat have been characterized, but of the 20 characterized 3 (NEATl(chrl:204.8), khpsla (chr10:103.4), Zfhx2as (chr15:31.8)) had an overlap with the ECR identified. Further analysis of the rat lncRNA is needed before future correlation with the ECR can be made. The ECR provide one explanation for how a limited number of DMR can potentially control a large number of differentially regulated genes. The locations of the potential ECR are included in Figure 8 to correlate with the DMR identified.

Previously the sperm DMR identified in vinclozolin lineage F3 generation animals was reported [16]. An overlap of these sperm DMR with the current granulosa cell DMR demonstrated no overlapped sites. The lack of DMR overlap demonstrates different transgenerational epigenomes between the sperm and granulosa cell. It is anticipated that the cascade of epigenetic and transcriptome steps to achieve a differentiated somatic cell will lead to very distinct cell specific epigenomes with minimal overlap with the germ line [14]. Therefore, observations demonstrate that the F3 generation vinclozolin lineage granulosa cells have transgenerational changes in the epigenome that correlate with transgenerational changes in the transcriptome that in turn are proposed to have a role in the induction of the transgenerational ovarian disease. All the other cell types in the ovary (e.g. oocyte, theca cells, ovarian stromal cells) are also expected to have transgenerational epigenome and transcriptome changes that will also contribute to ovarian disease. The granulosa cell observations provide the proof of concept that the transgenerational disease phenotype develops from the transgenerational effects of the altered epigenome on somatic cell transcriptomes.

\section{Discussion}

The most common human diseases of the ovary are primary ovarian insufficiency and polycystic ovarian disease. These conditions can cause infertility and increase the risk for other related health issues. Primary ovarian insufficiency affects about $1 \%$ of women, while polycystic ovarian disease affects as many as $18 \%$ of women $[25,26,27,37,38,39,40,41,42,44]$. In the current study, F0 generation gestating female rats were exposed to various environmental compounds during fetal gonadal sex determination followed by F1 and F3 generation progeny being examined for ovarian histology. Ovarian abnormalities resembling the follicle pool depletion that precedes primary ovarian insufficiency and the cyst formation of polycystic ovarian disease were observed transgenerationally at an increased rate in the F3 generation exposure lineage animals. Molecular studies were performed comparing F3 generation control to vinclozolin lineage animals that indicated that there were transgenerational alterations in the epigenome and transcriptome of granulosa cells from ovarian follicles. These results raise the possibility that the disease etiology may in part be a result of exposure to environmental toxicants that promote epigenetic transgenerational inheritance of ovarian disease.

The analysis of ovarian follicle counts showed that there were significantly fewer oocytes in the ovaries of all of the exposure lineage females. Mean decreases in primordial follicle counts of $35 \%$ to $60 \%$ were seen in both the $\mathrm{F} 1$ and the $\mathrm{F} 3$ generation animals (Fig. 1). Since Fl generation animals were directly exposed to the environmental compounds in utero when the F0 gestating females were exposed, the F1 generation decrease in oocyte number compared to controls can be due to direct exposure of the follicles to the compounds. This possibility was tested using an organ culture system in which ovaries isolated from neonatal rats were treated with varying doses of vinclozolin or were left untreated as controls. A dose of $500 \mu \mathrm{M}$ vinclozolin resulted in significantly fewer oocytes, while $200 \mu \mathrm{M}$ and lower concentrations were not significantly different from controls (Fig. 2). F0 generation gestating female rats were treated with $100 \mathrm{mg} / \mathrm{kg}$ vinclozolin, which converts to approximately a $350 \mu \mathrm{M}$ dose (assuming a whole-body volume of distribution). So it is conceivable that germ cells/oocytes could be lost in the F1 females when their F0 generation mothers are treated with vinclozolin. The epigenetic transgenerational inheritance of adult onset disease induced by the toxicants used in previous studies $[19,78]$ demonstrates that the compounds or their metabolites pass the placenta to reach the fetus. Direct exposure to several of the toxicants used in this study has previously been shown to affect 


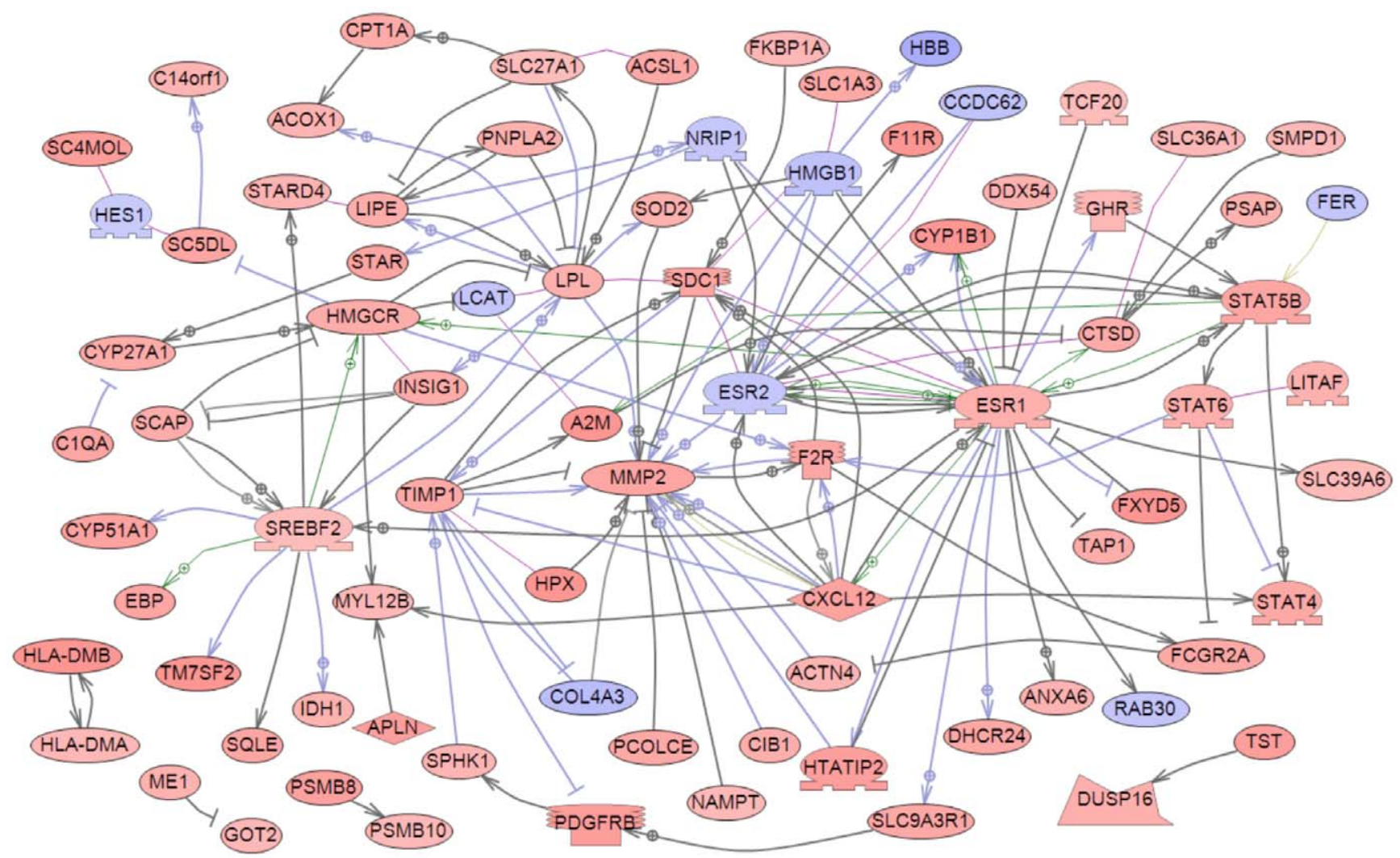

Figure 6. Gene network of known relationships among those genes found to be differentially expressed in Control compared to Vinclozolin-lineage F3 generation granulosa cells. Network is derived from an un-biased search of literature using Pathway Studio ${ }^{\text {TM }}$. Node shapes code: oval and circle - protein; diamond - ligand; irregular polygon - phosphatase; circle/oval on tripod platform - transcription factor; ice cream cone - receptor. Red color represents up-regulated genes, blue color - down-regulated genes, grey rectangles represent cell processes; arrows with plus sign show positive regulation/activation, arrows with minus sign - negative regulation/inhibition. Grey arrows represent regulation, lilac expression, green - promoter binding, olive - protein modification.

doi:10.1371/journal.pone.0036129.g006

ovarian function and development. Neonatal exposure to BPA has been shown to decrease the pool of primordial follicles and increase the incidence of ovarian cyst formation in rat ovaries, similarly to results of the current study $[79,80]$. Exposure to the plastic phthalate DEHP was shown to inhibit steroidogenesis in rat granulosa cells [81], and to impair oocyte growth and ovulation in zebrafish [82]. Female rats treated with dioxin both during fetal development and after birth were reported to have a premature cessation of reproductive cycles as they aged [65]. However, these direct exposure effects cannot explain the significant decrease in oocyte number seen in F3 generation exposure lineage females. F3 generation females were not directly exposed to the environmental compounds, which suggests a potential epigenetic transgenerational inheritance molecular mechanism.

Primary ovarian insufficiency in humans is characterized by an early loss of ovarian follicles and onset of menopause. This can occur when the pool of oocytes in the ovary is depleted to less than $1 \%$ of the amount present at puberty, as occurs with menopause $[83,84]$. The major physiological parameter of POI is the loss of the primordial follicle pool. In the current study, F1 and F3 treated-lineage groups of animals showed a 35-60\% decrease in primordial follicle numbers compared to age-matched one-yearold controls. Approximately $45 \%$ of all the exposure lineage F3 generation females developed follicle pool depletion. Normal female rats usually stop cycling and enter reproductive senescence at 15-18 months of age. Other studies have shown that experimental depletion of oocyte numbers in rodents leads to an early loss of reproductive cycles [85,86,87]. Therefore, it is expected that animals from toxicant exposure lineages with follicle pool depletion would have a higher incidence of premature reproductive senescence (e.g. POI), but this remains to be investigated.

Polycystic ovarian disease is characterized by multiple persistent ovarian cysts [43]. In the current study increased numbers of ovarian cysts were seen in all the treated-lineage groups compared to the control lineage groups (Fig. 3). Interestingly, this effect was much more pronounced in the transgenerational F3 animals than in the directly exposed F1 generation. This suggests that the PCO disease identified may be due primarily to epigenetic transgenerational mechanisms and not to direct exposure. In addition, $\mathrm{PCO}$ disease was primarily observed in the 1 year old animals and not in young adults of 120 days of age [19], which is similar to what is observed in humans. An increase in circulating androstenedione was observed in the F3 generation vinclozolin lineage females that had PCO, similar to the clinical phenotype in women with PCO. Interestingly, research has shown that androstenedione levels are also increased in animals with follicle pool depletion [88]. The large cysts found in environmental exposure lineage females (vinclozolin, pesticide, jet-fuel and low-dose plastics groups) often had a negligible layer of epithelial/granulosa cells lining the cavity and only a stromal/thecal layer surrounding the cyst. These resemble the follicular cysts in PCOS patients [89,90,91]. 


\section{(A) Ovarian Disease Associated Genes}

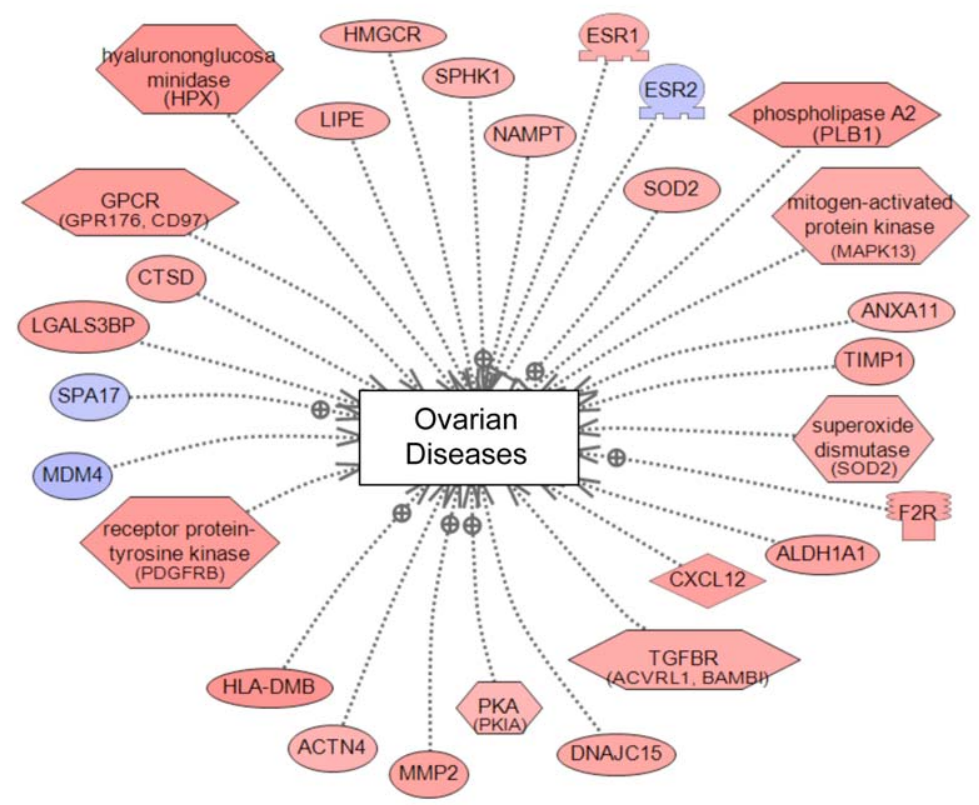

\section{(B) Polycystic Ovarian Disease Associated Genes}

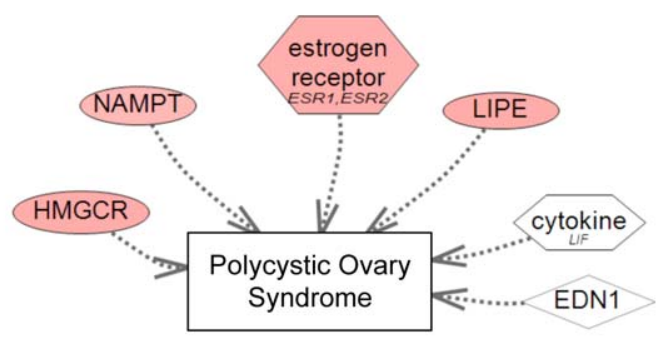

Figure 7. Ovarian diseases associated differentially expressed genes between F3 generation vinclozolin and control lineage granulosa cell. Sub-networks were identified using global literature analysis with Pathway Studio ${ }^{\mathrm{TM}}$. Node and arrow code is the same as for Figure 6. A: General ovarian diseases associated genes. B: Polycystic ovarian disease associated genes. White color nodes indicate differential methylated regions identified in this study.

doi:10.1371/journal.pone.0036129.g007

Luteal cysts were found only in the jet-fuel exposure lineage animals. These are cysts thought to form in the center of corpora lutea and are characterized by their surrounding band of luteal cells. This finding suggests that treatment with the different environmental toxicants can result in different transgenerational phenotypes. In contrast, all the different toxicant treatments resulted in the same increase in small ovarian cysts and in the same decrease in the primordial follicle pool. This is the case even though the different environmental exposures used are chemically dissimilar and would be expected to act through different signaling mechanisms. Observations suggest that some physiological processes in the ovary may be more prone to dys-regulation, independent of the environmental insult. For example, the complex signaling network that maintains primordial follicles and their oocytes in an arrested state [70,74,92,93] may be sensitive to dys-regulation that then leads to accelerated loss of follicles and oocytes from the ovary. Further research into this environmentally induced epigenetic transgenerational inheritance model is needed to determine the specific etiologies of adult onset ovarian diseases.

\section{Molecular Etiology of Transgenerational Adult Onset Ovarian Disease}

All the environmental exposures used in the current study induced transgenerational ovarian abnormalities. From among these exposures vinclozolin was used as a toxicant to study the molecular changes occurring transgenerationally in granulosa cells. Although all ovarian cell types (e.g. oocytes) are anticipated to develop a transgenerational alteration in the transcriptome and epigenome, granulosa cells were selected to provide the proof of concept for this phenomena. Vinclozolin is an agricultural fungicide with anti-androgenic endocrine disrupting activity [94]. Several studies have shown transgenerational effects following exposure of gestating rats during the period of fetal gonadal sex determination to vinclozolin [13]. These effects in the F3 generation animals include increased incidence of adult onset diseases such as cancer, kidney disease, immune abnormalities, prostate disease, spermatogenic defects and infertility $[20,75,78,95,96]$. F3 generation female rats after ancestral vinclozolin exposure have been shown to have uterine bleeding abnormalities late in pregnancy [75]. The molecular mechanism 


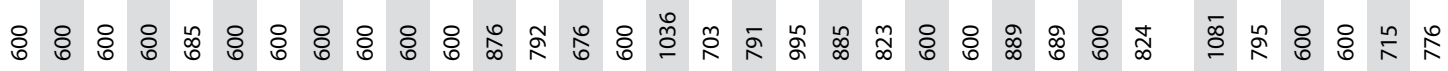

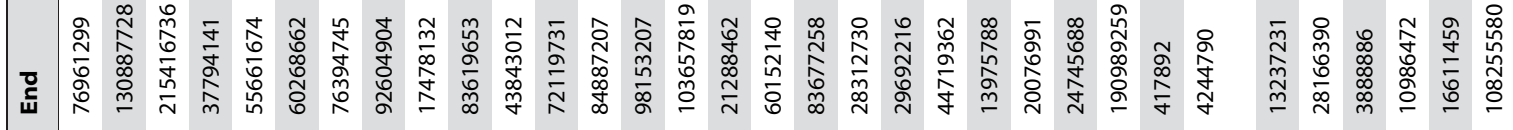

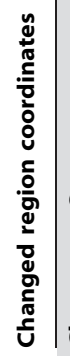

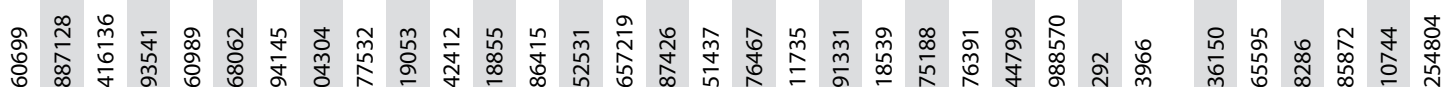

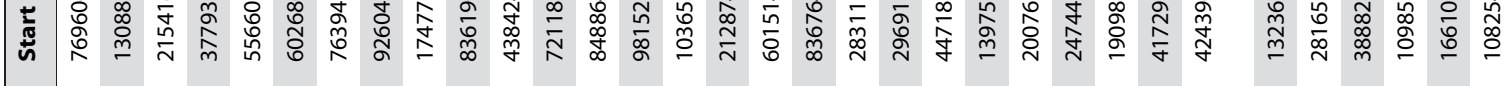

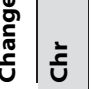

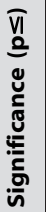

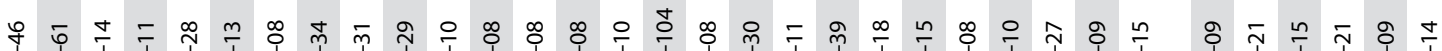

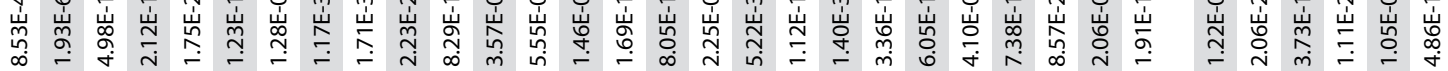

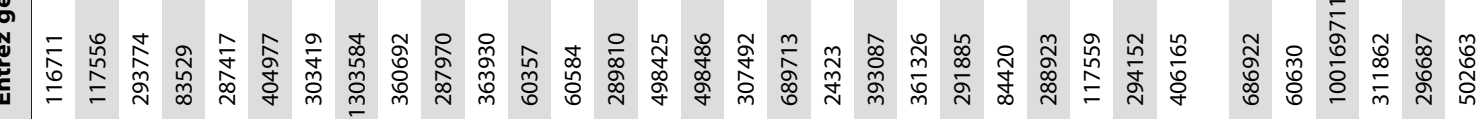

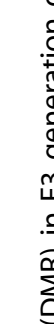

.

.

章

\section{党}

垔

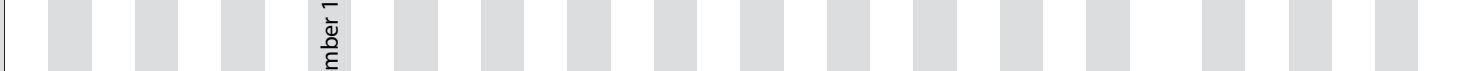

$\stackrel{m}{\leq}$

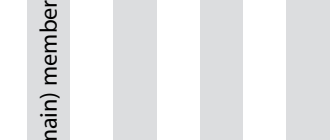

$a \quad \frac{\frac{\pi}{\pi}}{\frac{0}{0}}$

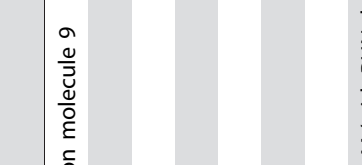

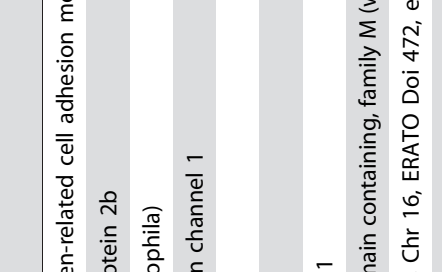

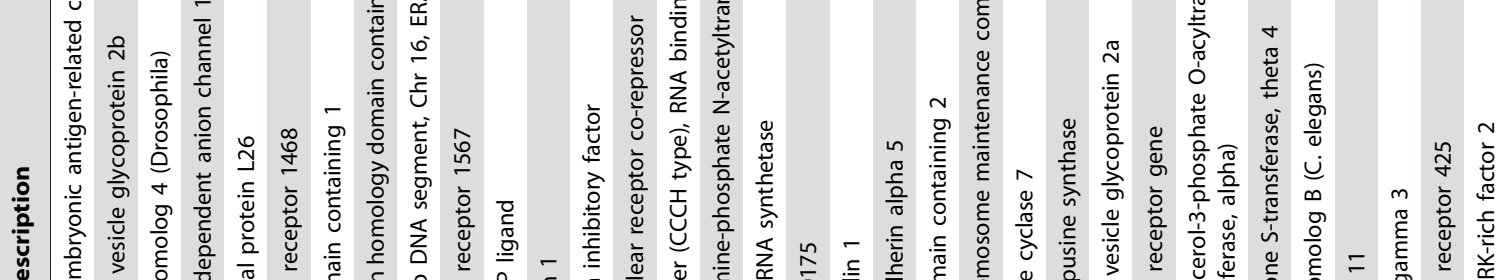

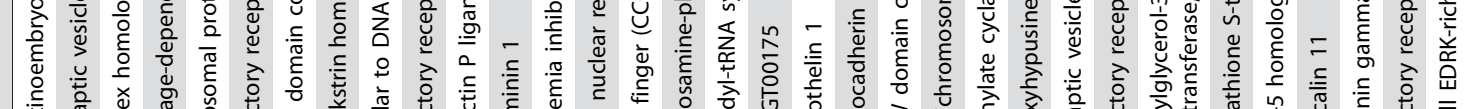

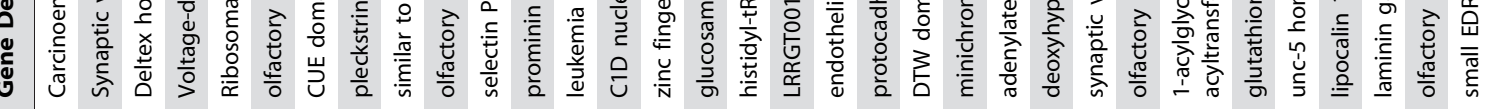

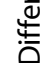




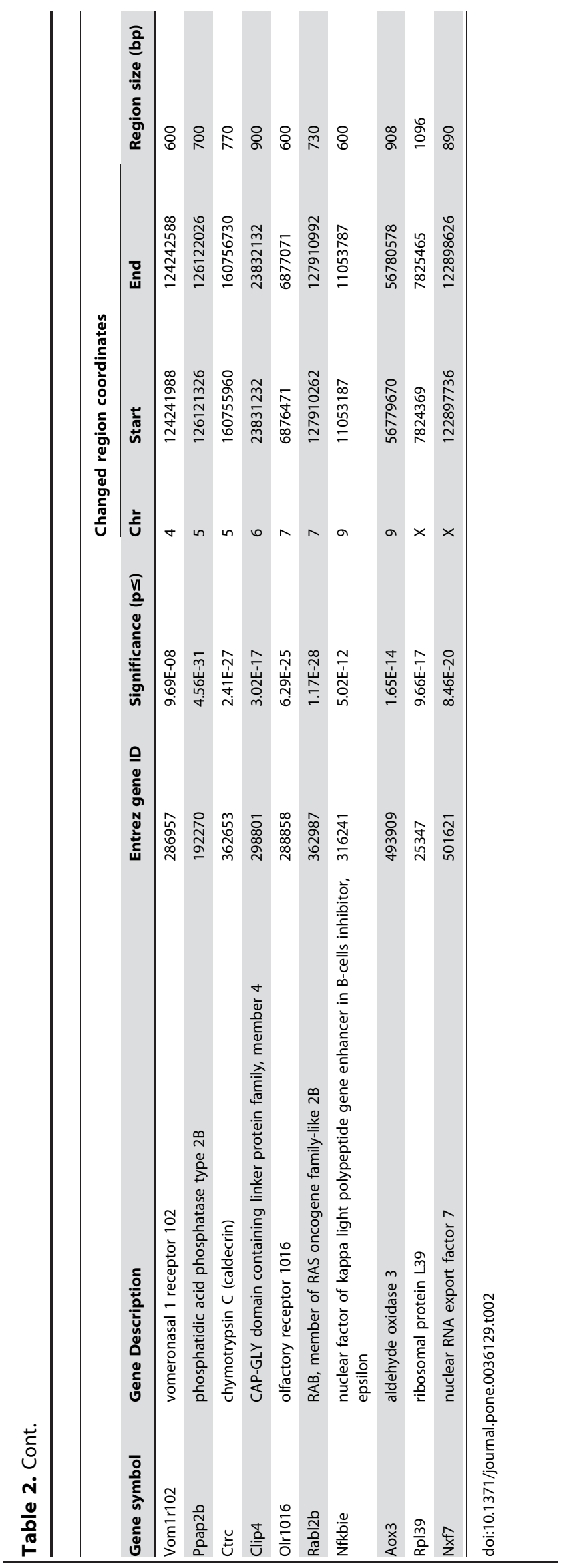

involved in epigenetic transgenerational inheritance requires an epigenetic alteration in the germline (egg or sperm) to transmit the phenotype [13]. An environmental exposure during fetal gonad sex determination appears to be required due to the epigenetic programming of primordial germ cells during this developmental period. Previous research with vinclozolin lineage rats has shown that permanent alterations in the male germ line epigenome are transmitted to subsequent generations and do not get erased after fertilization during early embryonic development, in a manner similar to imprinted genes [16]. A recent study demonstrates all the exposures used in the current study promote exposure specific epigenetic transgenerational alterations in the sperm epigenome [19]. Since the altered base-line epigenome of the sperm promotes an altered epigenome in cells and tissues that develop from that sperm, all tissues, including the ovary, are anticipated to have altered genome activity and develop a susceptibility to develop disease [76].

In the current study granulosa cells from large antral follicles of F3 generation females were evaluated for differences in either the gene expression profile or the epigenetic pattern of vinclozolinlineage granulosa compared to controls. The gene expression of granulosa cells from F3 generation vinclozolin and control lineage animals was evaluated by microarray analysis. More than 500 genes were found to be differentially expressed compared to controls (Table S1). This is a transgenerational effect of the environmental compound exposure. The altered gene expression profile of vinclozolin lineage granulosa cells could contribute to the adult-onset development of abnormalities such as primary ovarian insufficiency or polycystic ovarian disease. Bioinformatic analysis of the differentially expressed gene list indicated that certain wellcharacterized cellular pathways and processes could be affected by changes in these genes (Table 1, Figure S2a, S2b). Interestingly, many genes involved in lipid metabolism and steroid precursor synthesis had altered expression, and this has been shown to potentially be involved in the pathology of polycystic ovarian disease [43]. Analysis of genes present within the 523 differentially expressed gene set that have previously been correlated to ovarian disease revealed 30 genes, Figure 7. In addition, 5 genes have been shown to be directly correlated to polycystic ovarian disease. Therefore, the current study involving an environmental toxicant induced epigenetic transgenerational inheritance of adult onset ovarian disease also identified a number of genes previously shown to be associated with ovarian disease. A previous study compared the transcriptomes of cumulus granulosa cells from human PCOS patients and normal control women after culturing the cumulus cells. Similarly to the current study, genes in the MAPK signaling pathway and in extracellular matrix/cell adhesion were found to be affected. However, few specific differentially expressed genes were found in common with the current study [97].

A gene network analysis of the transgenerationally altered granulosa cell transcriptome generated a highly connected set of potential regulatory genes (Fig. 6) associated with the ovarian abnormalities identified. This regulatory gene network provides potential new therapeutic targets and diagnostic markers to consider in ovarian disease etiology. Critical gene targets for future studies to be considered include Esr1, Esr2, Srebf2, Mmp2, Cxcl12, Lpl, Stat5b and Hmgcr.

The F3 generation granulosa cell epigenome analysis of differential DNA methylation demonstrated 43 different DMR in promoters. The MeDIP-Chip analysis used a comparative hybridization of F3 generation granulosa cell DNA for vinclozolin versus control lineage animals to increase sensitivity. A promoter tiling array Chip was used, so the majority of the genome was not examined. Therefore, the $43 \mathrm{DMR}$ identified in promoters are a 


\section{Granulosa Epigenome and Transcriptome Clusters}

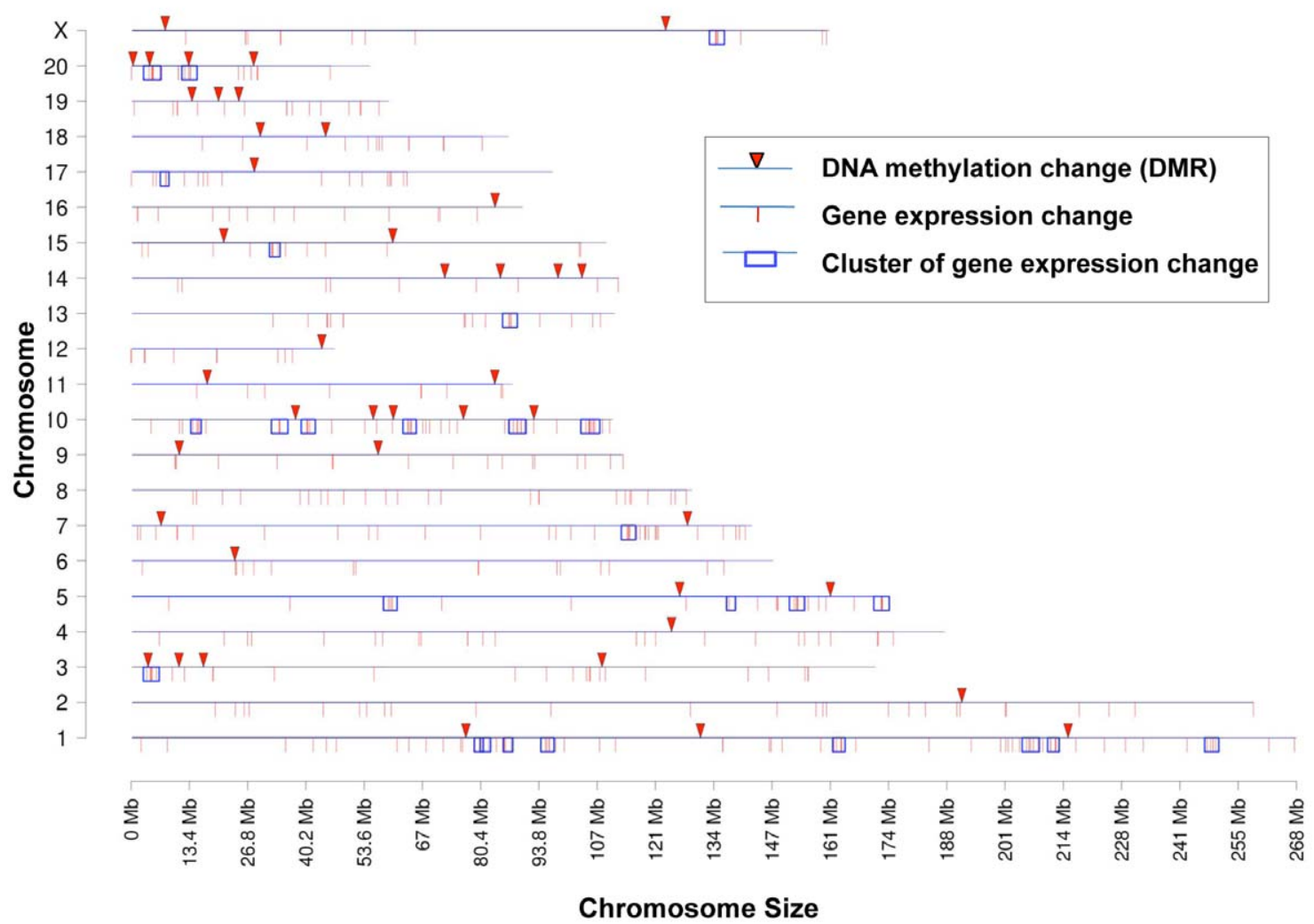

Figure 8. Chromosomal plot showing regions with vinclozolin-induced transgenerational changes in granulosa cells. Differential DNA methylation regions are displayed as inverted red triangles, changes in gene expression are displayed as red ticks, and a significant gene cluster of these genes with changed expression is delineated with blue open boxes. The chromosome number on $\mathrm{Y}$-axis and size on $\mathrm{X}$-axis are presented. doi:10.1371/journal.pone.0036129.g008

sub-set of the total epigenetic modifications possible. The anticipation is a larger set of epigenetic modifications are present genome wide. The 43 DMR identified in the F3 generation granulosa cell epigenome demonstrates an environmental induced transgenerational alteration that is correlated to the onset of ovarian abnormalities. The DMR were present on most autosomes. The CpG content of these DMR was 1-10 CpG per 100 bp. Previously, low density CpG regions have been shown to be involved in epigenetic transgenerational alterations in sperm $[19,98]$. This genomic feature is speculated to be important in the epigenetic programming mechanism [19]. Interestingly, only one DMR (Plekhm1) was present in the promoter of one of the transgenerational 523 differentially expressed granulosa cell genes. This is likely due to a random overlap. Therefore, a relatively low number of epigenetic DMR sites could not explain the relatively large number of differentially expressed genes observed. Direct epigenetic regulation of individual promoters appears not to be involved. The hypothesis developing is that the epigenetic regulatory sites associated with the DMR may influence distal gene expression through non-coding RNA and are termed epigenetic control regions (ECR). This is similar on a molecular level to the imprinting control regions (ICR) previously identified (e.g. IGF2 and H19) [77]. The transgenerational differentially expressed gene set of 523 genes was examined in regards to chromosomal location and 26 gene clusters of $2-5$ Mbase were identified with a statistically significant $(\mathrm{p}<0.05)$ over-represented set of genes, Figure 8 and Table S2. Several of these gene clusters correlated to the location of a DMR (approximately 15\%). In addition, the small number (i.e. 20) of characterized rat long noncoding RNA (lncRNA) had 3 sites that overlapped with the ECR, but further characterization of the rat lncRNA's is required before functional associations between ECR and lncRNA can be elucidated. Future studies will be needed to determine the functional significance of these potential ECR sites, but the current study suggests the potential presence of such sites. The potential presence of DMR regulating such an ECR is speculated to clarify how a limited number of alterations in the epigenome may influence a large number of differentially expressed genes.

The molecular factors involved in epigenetic regulation of genome activity (i.e. DNA methylation, histone modifications, chromatin structure and non-coding RNA) can all regulate proximal promoter activity and gene regulation. Epigenetic factors such as DNA methylation, chromatin structure and non-coding RNA can also regulate distal gene expression, independent of classic genetic mechanisms. In the current study environmentally induced transgenerational effects on the germ line promoted epigenome and transcriptome effects in the granulosa cell that correlate with adult onset ovarian abnormalities. The etiology of ovarian diseases such as PCO and POI appear to in part involve: 1) environmental toxicant induced epigenetic alterations in the germ-line (sperm) during fetal gonadal development; 2) permanent alterations in the epigenome that are transmitted to subsequent generations through the sperm; 3) induction of alterations in the epigenome and transcriptome of all organs, such as the ovary, and 
cells such as granulosa cells; and 4) an increased susceptibility to develop adult onset ovarian disease such as polycystic ovarian disease or primary ovarian insufficiency. Although the current study establishes the proof of concept such a mechanism exists, the degree the environment and epigenetic transgenerational inheritance is involved in human ovarian disease now needs to be investigated. Future studies are needed to clarify the F1, F2 and F3 generation sperm epigenome alterations in relation to each other, functional links of the DMR with the lncRNA and ECR, and to characterize transgenerational developmental changes in the transcriptome and epigenome of all ovarian cell types (e.g. oocyte, granulosa and theca). Elucidation of these molecular processes and mechanism will provide insights into the molecular etiology of ovarian disease.

\section{Summary}

An outbred rodent rat model was used to investigate the potential role of environmental epigenetics and epigenetic transgenerational inheritance in the etiology of ovarian disease. One of the ovarian abnormalities observed involved a decrease in the ovarian follicle pool size which correlates with the biology of primary ovarian insufficiency. The development of small and large ovarian cysts and the morphology of the cysts correlates with the biology of polycystic ovarian disease. However, these ovarian diseases as defined in humans are broader in concept to include correlated endocrine abnormalities and associated disease such as insulin resistance and diabetes. Therefore, the current ovarian abnormalities and disease in rats cannot be directly correlated to the human polycystic ovarian syndrome (PCOS) nor human primary ovarian insufficiency (POI) and loss of fertility. Although the rat ovarian abnormalities are consistent with these disease states, further research will be needed to clarify the role of environmental epigenetics and epigenetic transgenerational inheritance of ovarian disease in humans. Elucidation of such a disease etiology could help provide insight into clarifying the rapid increase in incidence of ovarian disease and apparent environmental impacts.

The environmental toxicants vinclozolin, dioxin and bisphenolA have been shown in previous research to have transgenerational effects $[21,22,75,78]$. In recent research from our laboratory, all of the environmental toxicants used in the current study were shown to cause transgenerational disease in rats [19]. In the current study DEHP and DBP were used in combination with bisphenol-A as a single treatment, so it is uncertain if alone the compounds can promote transgenerational disease in ovaries. Similarly, permethrin was used in combination with DEET, so evidence suggests the mixture can promote epigenetic transgenerational disease. The hydrocarbon mixture jet fuel (JP8) also promoted a transgenerational increase in the incidence of ovarian disease in these studies. Results suggest that all these compounds should now be considered as potentially able to promote transgenerational ovarian disease.

The current study used pharmacologic doses of all the compounds and mixtures based on approximately $1 \%$ of the oral LD50 dose for most compounds, Table S3. The objective was to determine if these exposures have the capacity to promote epigenetic transgenerational inheritance of a disease phenotype, and not to do risk assessment of the exposures. Now that the current study has established the transgenerational actions of these compounds, risk assessment toxicology involving dose curves of relevant environmental doses are needed. In addition to considering the mode of administration and dose, the critical window of exposure to promote the epigenetic transgenerational phenotype (gonadal sex determination) needs to be considered, which for the human is 6-18 weeks of gestation. The gestating women in the first half of pregnancy would be the population most sensitive to environmentally induced epigenetic transgenerational inheritance of disease phenotypes.

In summary, gestating F0 generation rats were treated with environmental toxicants transiently during fetal gonadal sex determination. Adult-onset ovarian diseases resembling primary ovarian insufficiency and polycystic ovarian disease were seen at an increased rate in both the directly exposed offspring (F1), and transgenerationally (F3). There was a significant transgenerational alteration in both the transcriptome and the epigenome of vinclozolin-lineage granulosa cells. Therefore, ancestral toxicant exposure can contribute to the development of these disease states. These results suggest a new paradigm be considered for the etiology of ovarian disease. In addition to genetic abnormalities being causative, epigenetic abnormalities can also cause changes in gene expression during development that lead to these adult-onset diseases. These epigenetic abnormalities can be induced by exposure to a variety of environmental toxicants. If the exposure occurs during a susceptible period of an animal's development, then these epigenetic abnormalities can be fixed into the germ line (i.e. eggs or sperm) and be passed transgenerationally. Ovarian disease such as PCO has impacts on other diseases such as diabetes and adverse pregnancy outcomes [99]. Therefore, further elucidation of the etiology of ovarian disease and potential role of environmental epigenetics and epigenetic transgenerational inheritance will provide insights into the prevention and therapeutic strategies for female health.

\section{Methods}

\section{Animals and treatments}

All experimental protocols involving rats were pre-approved by the Washington State University Animal Care and Use Committee (IACUC approval \# 02568-026). Hsd:Sprague Dawley ${ }^{\circledR \mathrm{TM}} \mathrm{SD}^{\circledR \mathrm{TM}}$ female and male rats of an outbred strain (Harlan) were maintained in ventilated (up to 50 air exchanges/hour) isolator cages (cages with dimensions of $10 \quad 3 / 4^{\prime \prime} \mathrm{W} \times 19 \quad 1 / 4^{\prime \prime} \mathrm{D} \times 10$ $3 / 4^{\prime \prime} \mathrm{H}, 143$ square inch floor space, fitted in Micro-vent 36-cage rat racks; Allentown Inc., Allentown, NJ) containing Aspen Sani chips (pinewood shavings from Harlan) as bedding, on a $14 \mathrm{~h}$ light: $10 \mathrm{~h}$ dark regimen, at a temperature of $70 \mathrm{~F}$ and humidity of $25 \%$ to $35 \%$. Rats were fed ad libitum with standard rat diet (8640 Teklad 22/5 Rodent Diet; Harlan) and ad libitum tap water for drinking.

At proestrus as determined by daily vaginal smears, the female rats (90 days of age) were pair-mated with male rats (120 days). On the next day, the pairs were separated and vaginal smears were examined microscopically. If sperm were detected (day 0 ) the rats were tentatively considered pregnant. Vaginal smears were continued for monitoring diestrus status in these rats until day 7 . Pregnant rats were then given daily intraperitoneal injections of any one of the following single chemicals or mixtures with an equal volume of sesame oil (Sigma) on days E-8 through E-14 of gestation [75], as seen in Table S3. Treatment groups were Control (DMSO vehicle), Vinclozolin, Pesticide/repellent (includes: Permethrin (insecticide) and DEET (insect repellent)), Plastics (Bisphenol-A, DBP and DEHP), Low-dose plastics, Dioxin (TCDD), and Jet Fuel (JP8 hydrocarbon). The pregnant female rats treated with various mixtures were designated as the F0 generation. A drop in litter size was noted in the F1 generation of the Plastics group, so another treatment group was included with only half the dose of Bisphenol-A, DBP and DEHP and this group was designated the 'Low Dose Plastics' group. Doses, percent of oral LD50, and sources of the compounds are given in Table S3. 


\section{Breeding for F1, F2, and F3 generations}

The offspring of the $\mathrm{F} 0$ generation were the $\mathrm{F} 1$ generation. The $\mathrm{F} 1$ generation offspring were bred to other $\mathrm{F} 1$ animals of the same treatment group to generate an F2 generation and then F2 generation animals bred similarly to generate the F3 generation animals. No sibling or cousin breedings were performed so as to avoid inbreeding. Note that only the original F0 generation pregnant females were injected with the treatment compounds.

\section{Evaluation of adult ovaries}

Ovaries taken from rats at the time of sacrifice (one year of age) were fixed in Bouin's solution, paraffin embedded and sectioned at $5 \mu \mathrm{m}$ thickness. Every $30^{\text {th }}$ section was collected and hematoxylin/ eosin stained. The three stained sections $(150 \mu \mathrm{m}$ apart) through the central portion of the ovary with the largest cross-section were evaluated microscopically for number of primordial follicles, developing pre-antral follicles, small antral follicles, large antral follicles, small cystic structures and large cysts. The mean number of each evaluated structure per section was calculated across the three sections. Follicles had to be non-atretic and have the oocyte nucleus visible in the section in order to be counted. Atretic follicles have granulosa cells or oocytes with pyknotic nuclei, an uneven or reduced layer of granulosa cells, and/or an uneven and less distinct basement membrane. Primordial follicles are in an arrested state and have an oocyte surrounded by a single layer of either squamous or both squamous and cuboidal granulosa cells $[100,101]$. Normally a few primordial follicles at a time will undergo primordial to primary follicle transition and become developing follicles. Developing pre-antral follicles had one or more complete layers of cuboidal granulosa cells. Small antral follicles had a fluid-filled antrum and a maximum diameter of $510 \mu \mathrm{m}$ measured across the outermost granulosa cell layer. Large antral follicles had a diameter greater than $510 \mu \mathrm{m}$. Large antral follicles may eventually ovulate. Cysts were defined as fluid-filled structures of a specified size that were not filled with red blood cells and which were not follicular antra. A single layer of cells may line cysts. Small cysts were $50-250 \mu \mathrm{m}$ in diameter measured from the inner cellular boundary across the longest axis. Large cysts were greater than $250 \mu \mathrm{m}$ in diameter.

\section{Neonatal rat ovary culture}

Four-day old female Sprague-Dawley rats (Harlan Laboratories, Inc., USA) were euthanized according to Washington State University IACUC approved protocols and their ovaries removed and cultured whole as described previously [102]. Four-day old rat ovaries contain follicles that are almost exclusively of the primordial stage. Whole ovaries were cultured on floating filters $(0.4 \mu \mathrm{m}$ Millicell-CM, Millipore, Bedford, MD, USA) in $0.5 \mathrm{ml}$ Dulbecco's modified Eagle's medium (DMEM)-Ham's F-12 medium (1:1, vol/vol) containing $0.1 \%$ BSA (Sigma), $0.1 \%$ Albumax (Gibco BRL, Gaithersburg, MD, USA), $27.5 \mu \mathrm{g} / \mathrm{ml}$ transferrin, and $0.05 \mathrm{mg} / \mathrm{ml} \mathrm{L-ascorbic} \mathrm{acid} \mathrm{(Sigma)} \mathrm{in} \mathrm{a} \mathrm{four-well}$ culture plate (Nunc plate, Applied Scientific, South San Francisco, CA, USA) for ten days. Previous studies have shown that four-dayold ovaries cultured for ten days have good cell viability [103]. The medium was supplemented with penicillin and streptomycin to prevent bacterial contamination. Ovaries were randomly assigned to treatment groups, with 1-3 ovaries per floating filter per well. Culture medium was changed and wells were treated every two days with vinclozolin $(50 \mu \mathrm{M}, 100 \mu \mathrm{M}, 200 \mu \mathrm{M}$, or $500 \mu \mathrm{M})$, or were treated with $0.1 \% \mathrm{DMSO}$ as a vehicle control. After culture, ovaries were fixed in Bouin's fixative (Sigma) for two hours and then equilibrated in $70 \%$ ethanol. Ovaries were then embedded in paraffin, sectioned at $3 \mu \mathrm{m}$ and stained with hematoxylin/eosin for use in morphological analysis.

For each ovary the number of oocytes per section was counted and the counts were averaged across the two consecutive histological sections that had the largest ovarian cross section. The oocyte nucleus had to be visible for an oocyte to be counted. Normally, between 50 and 150 follicles were present in each crosssection.

Blood samples were collected, allowed to clot, centrifuged and serum samples stored for hormone assays. The androstenedione levels in serum were determined with a radio-immunoassay (RIA) performed by the Center for Reproductive Biology Assay Core at Washington State University.

\section{Super-ovulation and collection of granulosa cells}

F3 generation rats from both vinclozolin-treated and control lineages were treated with Pregnant Mare Serum Gonadotropin (Sigma cat, St. Louis, MO)(30 IU PMSG injected IP) at five to six months of age. Two days later animals were sacrificed and ovaries removed. The ovarian bursa and its adherent fat was removed from each ovary and the ovaries processed for granulosa cell collection [104]. The ovaries were suspended in the base medium used for all procedures was Ham's F-12 (Thermo Scientific). Following sequential 30 minute incubations at $37{ }^{\circ} \mathrm{C}$ in $6 \mathrm{mM}$ EGTA in F-12 (to decrease $\mathrm{Ca}^{2+}$ - mediated cell adhesion) and then $0.5 \mathrm{M}$ sucrose in F-12 (to increase osmotic pressure within follicles), ovaries were returned to F-12. Granulosa cells were released into the medium from antral follicles using 30-gauge needles and gentle pressure. Oocytes were removed by aspiration under a dissecting microscope. Granulosa cells from each rat were collected into $1.5 \mathrm{~mL}$ tubes, allowed to settle for 10 minutes and supernatant removed. $1.0 \mathrm{~mL}$ of Trizol ${ }^{\mathrm{TM}}$ (Invitrogen) was added to each sample, and then samples were stored at $-70^{\circ}$ until the time of RNA and DNA isolation.

\section{Microarray transcriptome analysis}

Messenger RNA was isolated from Trizol ${ }^{\mathrm{TM}}$ for each animal as per manufacturers protocol. Messenger RNA from four animals of the same treatment group were pooled to create three different pooled samples from each of the two treatment groups. The mRNA processing and hybridization were performed at the Genomics Core Laboratory, Center for Reproductive Biology, Washington State University, Pullman, WA using standard Affymetrix reagents and protocol. Briefly, mRNA was transcribed into cDNA with random primers, then cRNA was transcribed from the cDNA, and from that, single-stranded sense DNA was synthesized which was fragmented and labeled with biotin. Biotinlabeled fragmented ssDNA was then hybridized to the Rat Gene 1.0 ST microarrays containing more than 27,000 transcripts (Affymetrix, Santa Clara, CA, USA). Hybridized chips were scanned on an Affymetrix Scanner 3000. CEL files containing raw data were then pre-processed and analyzed with Partek Genomic Suite 6.5 beta software (Partek Incorporated, St. Louis, MO) using an RMA and GC-content adjusted algorithm (Figure S1). The signals from an average of 28 different probes for each transcript were compared to give a single value. Lists of differentially expressed genes for each treatment were generated using the following cut off criteria: signal ratio Treatment/Control $>1.20$ change, mean difference for un-logged signals between control and treatment $>10$, t-test $\mathrm{p}$-values $<0.05$ using an analysis correcting for organ culture date batch effects.

CEL files from this study have been deposited with the NCBI gene expression and hybridization array data repository (GEO, http://www.ncbi.nlm.nih.gov/geo, \#GSE 33423) and can be also 
accessed through www.skinner.wsu.edu. For gene annotation, Affymetrix annotation file RaGene1_0stv1.na31.rn4.transcript.csv was used unless otherwise specified.

To look for known functional relationships among the F3 generation differentially expressed genes identified above, KEGG pathways were interrogated using the http://www.genome.jp/ kegg/ website (Kyoto Encyclopedia for Genes and Genome, Kyoto University, Japan), and also using Pathway Express, a webbased tool freely available as part of the Onto-Tools website (http://vortex.cs.wayne.edu) [105].

To further look for known functional relationships among the F3 differentially expressed genes, an unbiased, automated survey of published literature was performed to determine which genes are functionally linked with respect to binding, up-regulation, down-regulation, etc. Global literature analysis of differentially expressed genes was performed using Pathway Studio software (Ariadne, Genomics Inc. Rockville MD), which performs an interaction analysis and builds sub-networks of genes and the cellular processes that connect them to each other.

Previous studies have demonstrated that microarray data are validated with quantitative PCR data [106,107]. Due to the presence of an average of 28 different oligonucleotide probes for each specific gene being used on the microarray versus only a single primer set for a gene in a quantitative PCR, the microarray is more effective at eliminating false positive or negative data and provides a more robust quantification of changes in gene expression.

\section{Methylated DNA immunoprecipitation (MeDIP)}

DNA was collected from the same granulosa cell Trizol ${ }^{\mathrm{TM}}$ preparations that were used for RNA isolation. The DNA Trizol $^{\mathrm{TM}}$ fractions from four animals of the same treatment group were pooled to create three different pooled DNA samples from each of the two treatment groups. These DNA samples were then used for methylated DNA immunoprecipitation (MeDIP). MeDIP was performed as follows: $6 \mu \mathrm{g}$ of genomic DNA was subjected to series of three 20 pulse sonications at $20 \%$ amplitude and the appropriate fragment size (200-1000 bp) was verified through $2 \%$ agarose gels; the sonicated genomic DNA was resuspended in $350 \mu \mathrm{l} \mathrm{TE}$ and denatured for $10 \mathrm{~min}$ at $95^{\circ} \mathrm{C}$ and then immediately placed on ice for $5 \mathrm{~min} ; 100 \mu \mathrm{l}$ of $5 \times$ IP buffer (50 mM Na-phosphate pH7, $700 \mathrm{mM} \mathrm{NaCl,} \mathrm{0.25 \%} \mathrm{Triton} \mathrm{X-}$ 100) was added to the sonicated and denatured DNA. An overnight incubation of the DNA was performed with $5 \mu \mathrm{g}$ of antibody anti-5-methylCytidine monoclonal from Diagenode S.A (Denville, $\mathrm{NJ}$ ) at $4^{\circ} \mathrm{C}$ on a rotating platform. Protein A/G beads from Santa Cruz (Santa Cruz, CA) were prewashed on PBS-BSA $0.1 \%$ and resuspended in $40 \mu \mathrm{l} 1 \times \mathrm{IP}$ buffer. Beads were then added to the DNA-antibody complex and incubated $2 \mathrm{~h}$ at $4^{\circ} \mathrm{C}$ on a rotating platform. Beads bound to DNA-antibody complex were washed 3 times with $1 \mathrm{ml} 1 \times$ IP buffer; washes included incubation for $5 \mathrm{~min}$ at $4^{\circ} \mathrm{C}$ on a rotating platform and then centrifugation at $6000 \mathrm{rpm}$ for $2 \mathrm{~min}$. Beads-DNA-antibody complex were then resuspended in $250 \mu \mathrm{l}$ digestion buffer (50 mM Tris HCl pH 8, $10 \mathrm{mM}$ EDTA, 0.5\% SDS) and $3.5 \mu \mathrm{l}$ of proteinase $\mathrm{K}(20 \mathrm{mg} / \mathrm{ml})$ was added to each sample and then incubated overnight at $55^{\circ} \mathrm{C}$ on a rotating platform. DNA purification was performed first with phenol and then with chloroform:isoamyl alcohol. Two washes were then performed with $70 \%$ ethanol, $1 \mathrm{M} \mathrm{NaCl}$ and glycogen. MeDIP selected DNA was then resuspended in $30 \mu \mathrm{l} \mathrm{TE}$ buffer. Whole-genome amplification was then performed with the WGA2 kit (SigmaAldrich \#WGA2) on each MeDIP sample to be used in the microarray comparative hybridization analysis.

\section{Tiling Array MeDIP-Chip Analysis}

Roche Nimblegen's Rat DNA Methylation 3x720K CpG Island Plus RefSeq Promoter Array was used, which contains three identical sub-arrays, with 713,670 probes per sub-array, scanning a total of 15,287 promoters (3,880 bp upstream and $970 \mathrm{bp}$ downstream from transcription start site). Probe sizes range from 50-75 mer in length with a median probe spacing of $100 \mathrm{bp}$. Three different comparative (amplified MeDIP vs. amplified MeDIP) hybridizations experiments (3 sub-arrays) were performed, each encompassing DNA samples from 24 animals (3 treatment and 3 control groups). MeDIP DNA samples from experimental groups were labeled with $\mathrm{Cy} 3$ and MeDIP DNA samples from the control groups were labeled with Cy5.

\section{Bioinformatic and Statistical Analyses of Chip Data}

For each comparative hybridization experiment, raw data from both the Cy3 and Cy5 channels were imported into $\mathrm{R}$ ( $\mathrm{R}$ Development Core Team (2010), R: A language for statistical computing, R Foundation for Statistical Computing, Vienna, Austria. ISBN 3-900051-07-0, URL http://www.R-project.org), checked for quality and converted to MA values $(\mathrm{M}=\mathrm{Cy} 5-\mathrm{Cy} 3$; $\mathrm{A}=(\mathrm{Cy} 5+\mathrm{Cy} 3) / 2)$. The following normalization procedure was conducted. Within each array, probes were separated into groups by GC content and each group was separately normalized, between $\mathrm{Cy} 3$ and Cy5 using the loess normalization procedure. This allowed for GC groups to receive a normalization curve specific to that group. After each array had its CG groups normalized within the array, the arrays were then normalized across arrays using the A quantile normalization procedure.

Following normalization each probe within each array was subjected to a smoothing procedure, whereby the probe's normalized $\mathrm{M}$ values were replaced with the median value of all probe normalized $\mathrm{M}$ values across all arrays within a $600 \mathrm{bp}$ window. If the number of probes present in the window was less than 3, no value was assigned to that probe. Each probe's A values were likewise smoothened using the same procedure. Following normalization and smoothing each probe's $\mathrm{M}$ value represents the median intensity difference between vinclozolin generation and control generation of a $600 \mathrm{bp}$ window. Significance was assigned to probe differences between treatment-generation samples and control generation samples by calculating the median value of the intensity differences as compared to a normal distribution scaled to the experimental mean and standard deviation of the normalized M. A Z-score and P-value were computed for each probe from that distribution. The statistical analysis was performed in pairs of comparative IP hybridizations between treatment lineage $(\mathrm{T})$ and control lineage $(\mathrm{C})$. T1-C1 and T2-C2 gave 333 sites; T1-C1 and T3-C3 gave 327 sites; T2-C2 and T3-C3 gave 340 sites. In order to assure the reproducibility of the candidates obtained, only the candidates showing significant changes in all three of the paired comparisons were chosen as having a significant change in DNA methylation between the experimental group and controls. This is a very stringent approach to select for changes, since it only considers those changes repeated in all paired analyses.

Clustered Regions of interest were then determined by combining consecutive probes within 600 bases of each other, and based on whether their mean $M$ values were positive or negative, with significance $\mathrm{p}$-values less than $10^{-5}$. The statistically significant differential DNA methylated regions were identified and $\mathrm{P}$-value associated with each region presented. Each region of interest was then annotated for gene and CpG content. This list was further reduced to those regions with an average intensity value exceeding 9.5 (log scale) and a CpG density $\geq 1$ CpG/ $100 \mathrm{bp}$. 
Statistical Analysis for ovarian morphological data

Treatment groups are compared using analysis of variance (ANOVA) followed by Dunnet's post-hoc tests where appropriate. Groups were considered statistically significant with $\mathrm{P} \leq 0.05$. Statistics for ovary counts were calculated using Graph Pad Prism version 5.0 b for Macintosh, Graph Pad Software, San Diego, CA, USA.

\section{Supporting Information}

Figure S1 Sample histograms and box plots for granulosa cell microarray signal values after pre-processing with RMA, GG-content adjusted algorithm. Plots for F3 generation control (red) and F3 generation vinclozolin (blue) microarrays.

(PDF)

Figure S2 (A): Steroid Biosynthesis Pathway; and (B): PPAR Signaling Pathway showing granulosa cell differentially expressed genes between $F 3$ generation vinclozolin and control lineage rats: red or red-countered boxes represent up-regulated genes, green down-regulated and white boxes - not affected genes.

(PDF)

\section{References}

1. Barker DJ (2007) The origins of the developmental origins theory. J Intern Med 261: 412-417.

2. Jirtle RL, Skinner MK (2007) Environmental epigenomics and disease susceptibility. Nat Rev Genet 8: 253-262.

3. Bale TL, Baram TZ, Brown AS, Goldstein JM, Insel TR, et al. (2010) Early life programming and neurodevelopmental disorders. Biol Psychiatry 68: 314-319.

4. Godfrey KM, Gluckman PD, Hanson MA (2010) Developmental origins of metabolic disease: life course and intergenerational perspectives. Trends Endocrinol Metab 21: 199-205.

5. Clayton EM, Todd M, Dowd JB, Aiello AE (2011) The impact of bisphenol A and triclosan on immune parameters in the U.S. population, NHANES 20032006. Environ Health Perspect 119: 390-396.

6. Hylkema MN, Blacquiere MJ (2009) Intrauterine effects of maternal smoking on sensitization, asthma, and chronic obstructive pulmonary disease. Proc Am Thorac Soc 6: 660-662.

7. Nuyt AM (2008) Mechanisms underlying developmental programming of elevated blood pressure and vascular dysfunction: evidence from human studies and experimental animal models. Clin Sci (Lond) 114: 1-17.

8. Kaati G, Bygren LO, Edvinsson S (2002) Cardiovascular and diabetes mortality determined by nutrition during parents' and grandparents' slow growth period. Eur J Hum Genet 10: 682-688.

9. Roghair RD, Lamb FS, Miller FJJ, Scholz TD, Segar JL (2005) Early gestation dexamethasone programs enhanced postnatal ovine coronary artery vascular reactivity. Am J Physiol Regul Integr Comp Physiol 288: R46-53.

10. Painter RC, Osmond C, Gluckman P, Hanson M, Phillips DI, et al. (2008) Transgenerational effects of prenatal exposure to the Dutch famine on neonatal adiposity and health in later life. BJOG 115: 1243-1249.

11. Brouwers MM, Feitz WF, Roelofs LA, Kiemeney LA, de Gier RP, et al. (2006) Hypospadias: a transgenerational effect of diethylstilbestrol? Hum Reprod 21: 666-669

12. Susiarjo M, Hassold TJ, Freeman E, Hunt PA (2007) Bisphenol A exposure in utero disrupts early oogenesis in the mouse. PLoS Genet 3: e5.

13. Skinner MK, Manikkam M, Guerrero-Bosagna C (2010) Epigenetic transgenerational actions of environmental factors in disease etiology. Trends Endocrinol Metab 21: 214-222.

14. Skinner MK (2011) Role of epigenetics in developmental biology and transgenerational inheritance. Birth Defects Res C Embryo Today 93: 51-55.

15. Skinner MK (2008) What is an epigenetic transgenerational phenotype? F3 or F2. Reprod Toxicol 25: 2-6.

16. Guerrero-Bosagna C, Settles M, Lucker BJ, Skinner MK (2010) Epigenetic transgenerational actions of vinclozolin on promoter regions of the sperm epigenome. PLoS ONE 5: e13100.

17. Thornburg KL, Shannon J, Thuillier P, Turker MS (2010) In utero life and epigenetic predisposition for disease. Adv Genet 71: 57-78.

18. Anway MD, Skinner MK (2008) Epigenetic programming of the germ line: effects of endocrine disruptors on the development of transgenerational disease. Reprod Biomed Online 16: 23-25.

19. Manikkam M, Guerrero-Bosagna C, Tracey R, Haque MM, Skinner MK (2012) Transgenerational Actions of Environmental Compounds on Repro-
Table S1 Rat granulosa cell genes differentially expressed between $F 3$ generation vinclozolin and control lineage animals (523 genes).

(PDF)

Table S2 Differential expressed gene clusters. (PDF)

Table S3 Doses and sources of chemicals used. (PDF)

\section{Acknowledgments}

We thank the expert technical assistance of Ms. Rebecca Tracey, Ms. Renee Espinosa Najera, Ms. Jessica Shiflett, Ms. Chrystal Bailey, Ms. Colleen Johns, and Mr. Md. Haque. We thank Ms. Heather Johnson for assistance in preparation of the manuscript. We acknowledge the helpful advice of Dr. David Jackson and Dr. John Lewis, US Army Center for Environmental Health Research, Department of Defense (DOD), and the leadership at the DOD TATRG.

\section{Author Contributions}

Conceived and designed the experiments: MKS. Performed the experiments: EN GL MM CGB MIS. Analyzed the data: MKS EN GL MM CGB MIS. Wrote the paper: MKS EN. Edited the manuscript: MKS EN GL MM GGB MIS.

ductive Disease and Epigenetic Biomarkers of Ancestral Exposures. PLoS ONE 7: e31901.

20. Anway MD, Cupp AS, Uzumcu M, Skinner MK (2005) Epigenetic transgenerational actions of endocrine disruptors and male fertility. Science 308: $1466-1469$

21. Salian S, Doshi T, Vanage G (2009) Perinatal exposure of rats to Bisphenol A affects the fertility of male offspring. Life Sci 85: 742-752.

22. Bruner-Tran KL, Osteen KG (2011) Developmental exposure to TCDD reduces fertility and negatively affects pregnancy outcomes across multiple generations. Reprod Toxicol 31: 344-350.

23. Stouder C, Paoloni-Giacobino A (2010) Transgenerational effects of the endocrine disruptor vinclozolin on the methylation pattern of imprinted genes in the mouse sperm. Reproduction 139: 373-379.

24. Coulam CB, Adamson SC, Annegers JF (1986) Incidence of premature ovarian failure. Obstet Gynecol 67: 604-606.

25. Maclaran K, Panay N (2011) Premature ovarian failure. J Fam Plann Reprod Health Care 37: 35-42.

26. Dixit H, Rao L, Padmalatha V, Raseswari T, Kapu AK, et al. (2010) Genes governing premature ovarian failure. Reprod Biomed Online 20: 724-740.

27. Persani L, Rossetti R, Cacciatore C (2010) Genes involved in human premature ovarian failure. J Mol Endocrinol 45: 257-279.

28. Smith P, Steckler TL, Veiga-Lopez A, Padmanabhan V (2009) Developmental programming: differential effects of prenatal testosterone and dihydrotestosterone on follicular recruitment, depletion of follicular reserve, and ovarian morphology in sheep. Biol Reprod 80: 726-736.

29. Abbott DH, Tarantal AF, Dumesic DA (2009) Fetal, infant, adolescent and adult phenotypes of polycystic ovary syndrome in prenatally androgenized female rhesus monkeys. Am J Primatol 71: 776-784.

30. Cordts EB, Christofolini DM, Dos Santos AA, Bianco B, Barbosa CP (2011) Genetic aspects of premature ovarian failure: a literature review. Arch Gynecol Obstet 283: 635-643

31. Knauff EA, Franke L, van Es MA, van den Berg LH, van der Schouw YT, et al. (2009) Genome-wide association study in premature ovarian failure patients suggests ADAMTS19 as a possible candidate gene. Hum Reprod 24: 2372-2378.

32. McGuire MM, Bowden W, Engel NJ, Ahn HW, Kovanci E, et al. (2011) Genomic analysis using high-resolution single-nucleotide polymorphism arrays reveals novel microdeletions associated with premature ovarian failure. Fertil Steril 95: 1595-1600

33. Zhao Z, Qin Y, Ma J, Zhao H, Li J, et al. (2011) PTEN gene analysis in premature ovarian failure patients. Acta Obstet Gynecol Scand 90: 678-679.

34. Persani L, Rossetti R, Cacciatore C, Fabre S (2011) Genetic defects of ovarian TGF-beta-like factors and premature ovarian failure. J Endocrinol Invest 34 : 244-251.

35. Pyun JA, Kang H, Kim J, Cha DH, Kwack K (2011) Thyroglobulin gene is associated with premature ovarian failure. Fertil Steril 95: 397-400.

36. Wang B, Suo P, Chen B, Wei Z, Yang L, et al. (2011) Haplotype analysis of chemokine CXCL12 polymorphisms and susceptibility to premature ovarian failure in Chinese women. Hum Reprod 26: 950-954. 
37. Woods KS, Reyna R, Azziz R (2002) Effect of oral micronized progesterone on androgen levels in women with polycystic ovary syndrome. Fertil Steril 77: 1125-1127.

38. Hart R, Hickey M, Franks S (2004) Definitions, prevalence and symptoms of polycystic ovaries and polycystic ovary syndrome. Best Pract Res Clin Obstet Gynaecol 18: 671-683.

39. Jones GL, Palep-Singh M, Ledger WL, Balen AH, Jenkinson C, et al. (2010) Do South Asian women with PCOS have poorer health-related quality of life than Caucasian women with PCOS? A comparative cross-sectional study. Health Qual Life Outcomes 8: 149.

40. Moran C, Tena G, Moran S, Ruiz P, Reyna R, et al. (2010) Prevalence of polycystic ovary syndrome and related disorders in mexican women. Gynecol Obstet Invest 69: 274-280.

41. Azziz R, Woods KS, Reyna R, Key TJ, Knochenhauer ES, et al. (2004) The prevalence and features of the polycystic ovary syndrome in an unselected population. J Clin Endocrinol Metab 89: 2745-2749.

42. March WA, Moore VM, Willson KJ, Phillips DI, Norman RJ, et al. (2010) The prevalence of polycystic ovary syndrome in a community sample assessed under contrasting diagnostic criteria. Hum Reprod 25: 544-551.

43. Rotterdam group (2004) Rotterdam ESHRE/ASRM-Sponsored PCOS consensus workshop group, Revised 2003 consensus on diagnostic criteria and long-term health risks related to polycystic ovary syndrome (PCOS). Hum Reprod 19: 41-47.

44. Glintborg D, Andersen M (2010) An update on the pathogenesis, inflammation, and metabolism in hirsutism and polycystic ovary syndrome. Gynecol Endocrinol 26: 281-296.

45. Franks S, McCarthy MI, Hardy K (2006) Development of polycystic ovary syndrome: involvement of genetic and environmental factors. Int J Androl 29: 278-285; discussion 286-290.

46. Chen ZJ, Shi Y (2010) Polycystic ovary syndrome. Front Med China 4: 280-284.

47. van Houten EL, Kramer P, McLuskey A, Karels B, Themmen AP, et al. (2012) Reproductive and Metabolic Phenotype of a Mouse Model of PCOS. Endocrinology.

48. Yanes LL, Romero DG, Moulana M, Lima R, Davis DD, et al. (2011) Cardiovascular-renal and metabolic characterization of a rat model of polycystic ovary syndrome. Gend Med 8: 103-115.

49. Manikkam M, Steckler TL, Welch KB, Inskeep EK, Padmanabhan V (2006) Fetal programming: prenatal testosterone treatment leads to follicular persistence/luteal defects; partial restoration of ovarian function by cyclic progesterone treatment. Endocrinology 147: 1997-2007.

50. Mukherjee S, Maitra A (2010) Molecular \& genetic factors contributing to insulin resistance in polycystic ovary syndrome. Indian J Med Res 131: 743-760.

51. Dasgupta S, Sirisha PV, Neelaveni K, Anuradha K, Reddy AG, et al. (2010) Androgen receptor CAG repeat polymorphism and epigenetic influence among the south Indian women with Polycystic Ovary Syndrome. PLoS One 5: e12401.

52. Menke MN, Strauss JF, 3rd (2007) Genetic approaches to polycystic ovarian syndrome. Curr Opin Obstet Gynecol 19: 355-359.

53. Li Z, Huang H (2008) Epigenetic abnormality: a possible mechanism underlying the fetal origin of polycystic ovary syndrome. Med Hypotheses 70: 638-642.

54. Hickey TE, Legro RS, Norman RJ (2006) Epigenetic modification of the X chromosome influences susceptibility to polycystic ovary syndrome. J Clin Endocrinol Metab 91: 2789-2791.

55. Kuriakose JS, Miller RL (2010) Environmental epigenetics and allergic diseases: recent advances. Clin Exp Allergy 40: 1602-1610.

56. Pogribny IP, Ross SA, Wise C, Pogribna M, Jones EA, et al. (2006) Irreversible global DNA hypomethylation as a key step in hepatocarcinogenesis induced by dietary methyl deficiency. Mutat Res 593: 80-87.

57. Nan HM, Song YJ, Yun HY, Park JS, Kim H (2005) Effects of dietary intake and genetic factors on hypermethylation of the hMLH1 gene promoter in gastric cancer. World J Gastroenterol 11: 3834-3841.

58. Martino D, Prescott S (2011) Epigenetics and prenatal influences on asthma and allergic airways disease. Chest 139: 640-647.

59. Choong MK, Tsafnat G (2011) Genetic and Epigenetic Biomarkers of Colorectal Cancer. Clin Gastroenterol Hepatol April 29: (Epub ahead of print).

60. Perry AS, Watson RW, Lawler M, Hollywood D (2010) The epigenome as a therapeutic target in prostate cancer. Nat Rev Urol 7: 668-680.

61. Hakre S, Chavez L, Shirakawa K, Verdin E (2011) Epigenetic regulation of HIV latency. Curr Opin HIV AIDS 6: 19-24.

62. Kaminsky Z, Tochigi M, Jia P, Pal M, Mill J, et al. (2011) A multi-tissue analysis identifies HLA complex group 9 gene methylation differences in bipolar disorder. Mol Psychiatry [Epub ahead of print].

63. Kitchen LW, Lawrence KL, Coleman RE (2009) The role of the United States military in the development of vector control products, including insect repellents, insecticides, and bed nets. J Vector Ecol 34: 50-61.

64. Talsness CE, Andrade AJ, Kurivama SN, Taylor JA, vom Saal FS (2009) Components of plastic: experimental studies in animals and relevance for human health. Philos Trans R Soc Lond B Biol Sci 364: 2079-2096.

65. Jablonska O, Shi Z, Valdez KE, Ting AY, Petroff BK (2010) Temporal and anatomical sensitivities to the aryl hydrocarbon receptor agonist 2,3,7,8- tetrachlorodibenzo-p-dioxin leading to premature acyclicity with age in rats. Int J Androl 33: 405-412.

66. Birnbaum LS, Fenton SE (2003) Cancer and developmental exposure to endocrine disruptors. Environ Health Perspect 111: 389-394.

67. Ritchie G, Still K, Rossi J, 3rd, Bekkedal M, Bobb A, et al. (2003) Biological and health effects of exposure to kerosene-based jet fuels and performance additives. J Toxicol Environ Health B Crit Rev 6: 357-451.

68. Wong SS, Vargas J, Thomas A, Fastje C, McLaughlin M, et al. (2008) In vivo comparison of epithelial responses for S-8 versus JP-8 jet fuels below permissible exposure limit. Toxicology 254: 106-111.

69. Mattie DR, Sterner TR (2011) Past, present and emerging toxicity issues for jet fuel. Toxicol Appl Pharmacol 254: 127-132.

70. Skinner MK (2005) Regulation of primordial follicle assembly and development. Hum Reprod Update 11: 461-471.

71. Peters H, Byskov AG, Himelstein-Braw R, Faber M (1975) Follicular growth: the basic event in the mouse and human ovary. J Reprod Fertil 45: 559-566.

72. Hirshfield AN (1991) Development of follicles in the mammalian ovary. Int Rev Cytol 124: 43-101.

73. Binelli M, Murphy BD (2010) Coordinated regulation of follicle development by germ and somatic cells. Reprod Fertil Dev 22: 1-12.

74. Nilsson EE, Savenkova MI, Schindler R, Zhang B, Schadt EE, et al. (2010) Gene bionetwork analysis of ovarian primordial follicle development. PLoS ONE 5: el1637.

75. Nilsson EE, Anway MD, Stanfield J, Skinner MK (2008) Transgenerational epigenetic effects of the endocrine disruptor vinclozolin on pregnancies and female adult onset disease. Reproduction 135: 713-721.

76. Skinner MK (2011) Environmental epigenetic transgenerational inheritance and somatic epigenetic mitotic stability. Epigenetics 6: 838-842.

77. Wan LB, Bartolomei MS (2008) Regulation of imprinting in clusters: noncoding RNAs versus insulators. Adv Genet 61: 207-223.

78. Anway MD, Leathers G, Skinner MK (2006) Endocrine disruptor vinclozolin induced epigenetic transgenerational adult-onset disease. Endocrinology 147: 5515-5523.

79. Rodriguez HA Santambrosio N, Santamaria CG Munoz-de-Toro M, Luque EH (2010) Neonatal exposure to bisphenol A reduces the pool of primordial follicles in the rat ovary. Reprod Toxicol 30: 550-557.

80. Fernandez M, Bourguignon N, Lux-Lantos V, Libertun C (2010) Neonatal exposure to bisphenol a and reproductive and endocrine alterations resembling the polycystic ovarian syndrome in adult rats. Environ Health Perspect 118 : 1217-1222.

81. Svechnikova I, Svechnikov K, Soder O (2007) The influence of di-(2ethylhexyl) phthalate on steroidogenesis by the ovarian granulosa cells of immature female rats. J Endocrinol 194: 603-609.

82. Carnevali O, Tosti L, Speciale C, Peng C, Zhu Y, et al. (2010) DEHP impairs zebrafish reproduction by affecting critical factors in oogenesis. PLoS ONE 5: e10201.

83. Macklon NS, Fauser BC (1999) Aspects of ovarian follicle development throughout life. Horm Res 52: 161-170.

84. De Vos M, Devroey P, Fauser BC (2010) Primary ovarian insufficiency. Lancet 376: 911-921.

85. Lohff JC, Christian PJ, Marion SL, Hoyer PB (2006) Effect of duration of dosing on onset of ovarian failure in a chemical-induced mouse model of perimenopause. Menopause 13: 482-488.

86. Anzalone CR, Hong LS, Lu JK, LaPolt PS (2001) Influences of age and ovarian follicular reserve on estrous cycle patterns, ovulation, and hormone secretion in the Long-Evans rat. Biol Reprod 64: 1056-1062.

87. Durlinger AL, Kramer P, Karels B, de Jong FH, Uilenbroek JT, et al. (1999) Control of primordial follicle recruitment by anti-Mullerian hormone in the mouse ovary. Endocrinology 140: 5789-5796.

88. Acosta JI, Mayer LP, Braden BB, Nonnenmacher S, Mennenga SE, et al. (2010) The cognitive effects of conjugated equine estrogens depend on whether menopause etiology is transitional or surgical. Endocrinology 151: 3795-3804.

89. Jordan CD, Bohling SD, Charbonneau NL, Sakai LY (2010) Fibrillins in adult human ovary and polycystic ovary syndrome: is fibrillin-3 affected in PCOS? J Histochem Cytochem 58: 903-915.

90. Wickenheisser JK, Nelson-DeGrave VL, McAllister JM (2006) Human ovarian theca cells in culture. Trends Endocrinol Metab 17: 65-71.

91. Nestler JE, Jakubowicz DJ, de Vargas AF, Brik C, Quintero N, et al. (1998) Insulin stimulates testosterone biosynthesis by human thecal cells from women with polycystic ovary syndrome by activating its own receptor and using inositolglycan mediators as the signal transduction system. J Clin Endocrinol Metab 83: 2001-2005.

92. Reddy P, Zheng W, Liu K (2010) Mechanisms maintaining the dormancy and survival of mammalian primordial follicles. Trends Endocrinol Metab 21: 96-103.

93. Buratini J, Price CA (2011) Follicular somatic cell factors and follicle development. Reprod Fertil Dev 23: 32-39.

94. Kelce WR, Monosson E, Gamcsik MP, Laws SC, Gray LE (1994) Environmental hormone disruptors: evidence that vinclozolin developmental toxicity is mediated by antiandrogenic metabolites. Toxicol Appl Pharmacol 126: 276-285.

95. Anway MD, Memon MA, Uzumcu M, Skinner MK (2006) Transgenerational effect of the endocrine disruptor vinclozolin on male spermatogenesis. J Androl 27: 868-879. 
96. Anway MD, Skinner MK (2008) Transgenerational effects of the endocrine disruptor vinclozolin on the prostate transcriptome and adult onset disease. Prostate 68: 517-529.

97. Kenigsberg S, Bentov Y, Chalifa-Caspi V, Potashnik G, Ofir R, et al. (2009) Gene expression microarray profiles of cumulus cells in lean and overweightobese polycystic ovary syndrome patients. Mol Hum Reprod 15: 89-103.

98. Guerrero-Bosagna C, Settles M, Lucker B, Skinner M (2010) Epigenetic transgenerational actions of vinclozolin on promoter regions of the sperm epigenome. PLoS ONE 5: e13100.

99. Roos N, Kieler H, Sahlin L, Ekman-Ordeberg G, Falconer H, et al. (2011) Risk of adverse pregnancy outcomes in women with polycystic ovary syndrome: population based cohort study. BMJ 343: d6309.

100. Meredith S, Dudenhoeffer G, Jackson K (2000) Classification of small type B/ C follicles as primordial follicles in mature rats. J Reprod Fertil 119: 43-48.

101. Nilsson EE, Schindler R, Savenkova MI, Skinner MK (2011) Inhibitory actions of Anti-Mullerian Hormone (AMH) on ovarian primordial follicle assembly. PLoS ONE 6: e20087.
102. Dole G, Nilsson EE, Skinner MK (2008) Glial-derived neurotrophic factor promotes ovarian primordial follicle development and cell-cell interactions during folliculogenesis. Reproduction 135: 671-682.

103. Schindler R, Nilsson E, Skinner MK (2010) Induction of ovarian primordial follicle assembly by connective tissue growth factor CTGF. PLoS ONE 5: e12979.

104. Peters CA, Maizels ET, Hunzicker-Dunn M (1999) Activation of PKC delta in the rat corpus luteum during pregnancy. Potential role of prolactin signaling. J Biol Chem 274: 37499-37505.

105. Draghici S, Khatri P, Tarca AL, Amin K, Done A, et al. (2007) A systems biology approach for pathway level analysis. Genome Res 17: 1537-1545.

106. Kezele PR, Ague JM, Nilsson E, Skinner MK (2005) Alterations in the ovarian transcriptome during primordial follicle assembly and development. Biol Reprod 72: 241-255.

107. Shima JE, McLean DJ, McCarrey JR, Griswold MD (2004) The Murine Testicular Transcriptome: Characterizing Gene Expression in the Testis During the Progression of Spermatogenesis. Biol Reprod 71: 319-330. 